\title{
Towards a universal shear correction factor in filament stretching rheometry
}

\author{
F. P. A. van Berlo ${ }^{1}$ - R. Cardinaels ${ }^{1}$. G. W. M. Peters ${ }^{1}$ - P. D. Anderson ${ }^{1}$ (i) \\ Received: 5 February 2021 / Revised: 28 May 2021 / Accepted: 8 August 2021 / Published online: 13 September 2021 \\ (c) The Author(s) 2021
}

\begin{abstract}
Filament stretching rheometry is a prominent experimental method to determine rheological properties in extensional flow whereby the separating plates determine the extension rate. In literature, several correction factors that can compensate for the errors introduced by the shear contribution near the plates have been introduced and validated in the linear viscoelastic regime. In this work, a systematic analysis is conducted to determine if a material-independent correction factor can be found for non-linear viscoelastic polymers. To this end, a finite element model is presented to describe the flow and resulting stresses in the filament stretching rheometer. The model incorporates non-linear viscoelasticity and a radius-based controller for the plate speed is added to mimic the typical extensional flow in filament stretching rheometry. The model is validated by comparing force simulations with analytical solutions. The effects of the end-plates on the extensional flow and resulting force measurements are investigated, and a modification of the shear correction factor is proposed for the nonlinear viscoelastic flow regime. This shows good agreement with simulations performed at multiple initial aspect ratios and strain rates and is shown to be valid for a range of polymers with non-linear rheological behaviour.
\end{abstract}

Keywords Filament stretching rheometer $\cdot$ Shear correction factor $\cdot$ Viscoelasticity $\cdot$ Extensional viscosity $\cdot$ Numerical simulation

\section{Introduction}

During processing, polymers undergo complex flow histories, which in general consist of a combination of shear and extensional flow. Depending on the process, one or the other may dominate, for instance processes such as fiber spinning, film blowing or extrusion from a nozzle are largely dominated by extensional flow. Nevertheless, even a cursory examination of textbooks on rheology (Bird et al. 1987; Macosko 1994; Tanner 2000; Morrison 2001) shows that rheological characterizations are mostly performed in shear flow, whereas characterization in extensional flow is less common. However, this is changing rapidly in the last two decades, probably due to the development of several commercial extensional rheometers (Sentmanat 2003; Hodder

\section{P. D. Anderson}

p.d.anderson@tue.nl

1 Department of Mechanical Engineering, Polymer Technology, Eindhoven University of Technology, 5600 Eindhoven, MB The Netherlands and Franck 2005; Huang et al. 2016). Over the years, several measuring techniques have been developed for this purpose. Setups such as fiber spinning (Kase and Matsuo 1965), contraction flows in extrusion dies (Cogswell 1972) or opposed jets (Fuller et al. 1987) clearly have the disadvantage of a complex geometry, thereby entailing complicated data analysis procedures to separate shear and extensional contributions which limits the accuracy of these measurement techniques. On the other hand, stretching a material film or filament could directly result in biaxial or uniaxial extensional flow. In case of uniaxial extension, a falling mass was initially used to generate the stretching motion (Matta and Tytus 1990). Later, several research groups have built variations of the so-called filament stretching rheometer (Sridhar et al. 1991; Tirtaatmadja and Sridhar 1993; McKinley et al. 1999; Anna et al. 2001; Bach et al. 2003; Chellamuthu et al. 2011; Pepe et al. 2020). In this device, a cylindrical fluid sample is placed between two parallel disks, that are then pulled apart by moving one plate while a load cell connected to the other plate measures the generated force. The plate speed determines the extension rate. Despite the seemingly simple principle, it was soon realized that extracting 
accurate extensional viscosity data from filament stretching experiments brings along several challenges (Meissner et al. 1981; Tirtaatmadja and Sridhar 1993). In extensional flow, fluid elements undergo a rapid and large deformation which requires a large travelling range of the pistons or clamps to stretch the fluid up to a sufficiently large strain (Meissner et al. 1981). In addition, the surface tension of the fluid will cause necking of the filament thereby resulting in a non-homogeneous diameter and thus non-homogeneous extension rate throughout the filament (Kröger et al. 1992). Finally, in case of fluids with a low to moderate viscosity that can not be clamped, the fluid is pinned to the moving pistons, resulting in no-slip boundary conditions. Hence, the fluid in the transition region between the no-slip boundary condition and the uniaxially extending filament (with a homogeneous inwards radial velocity) undergoes a combination of shear and extensional flow, accompanied by radial gradients in pressure. The latter pressure contributes to the normal force on the pistons, and thereby results in an overestimation of the extensional viscosity at the start of the experiment. After a certain critical strain is reached, locally (at the middle of the sample) a uniaxial extensional flow develops and the extensional viscosity measured at the pistons converges to the theoretical pure uniaxial extensional viscosity (Spiegelberg et al. 1996).

To overcome the above-mentioned challenges, several improvements of the original filament stretching rheometers have been done. Different modifications of the pistons were implemented to reduce the deviations of uniaxial extension at the solid-liquid contact region, including fixing the sample with epoxy resin (Münstedt 1975), using grippers (Vinogradov et al. 1970) and designing plates that dynamically change their diameter while the extensional test is ongoing (Berg et al. 1994). By doing interrupted stretch experiments, it was also noticed that the error introduced by the non-homogeneous deformation at the end plates depends on the initial aspect ratio. Hence, performing a pre-stretch of the sample followed by a short waiting period to allow relaxation of generated stresses, before performing the actual test, allows to reduce these errors and has become common practice in extensional rheometry experiments (Ooi and Sridhar 1994). Thereby, the ability to reach a sufficiently large Hencky strain becomes even more important. Using rotary clamps or rolls to stretch the sample is an effective means of reducing the physical length of the apparatus and has led to the development of commercial extensional rheology add-ons for rotational rheometers such as the SER and EVF setups (Sentmanat 2003; Hodder and Franck 2005). However, in these devices, the sample crosssection is in general not circular or square, which can lead to deviations from uniaxial extension (Nielsen et al. 2009; Hassager et al. 2010). Besides this, even though the exponentially increasing velocity required to maintain a constant overall extension rate can be applied on the plates, necking of the filament results in a non-homogeneous deformation along the filament (Spiegelberg et al. 1996; Yao and McKinley 1998). For Newtonian fluids, the local extension rate in the thinnest region of the filament can be $50 \%$ larger than the applied value (Spiegelberg et al. 1996) whereas in fluids with a complex rheology the extension rate can vary significantly throughout the experiment (Yao and McKinley 1998). In initial studies, trial-and-error was used to obtain an exponential radius decrease of the midradius of the filament (e.g. Tirtaatmadja and Sridhar 1993). Moreover, a master curve approach was introduced by Orr and Sridhar (1999) to determine the optimal velocity profile of the plates, from which an iterative approach was developed for polymer melts (Bach et al. 2003). Later, several control schemes were implemented that use feedback and feed forward control combined with in situ measurements of the filament diameter to ensure a constant uniaxial extension rate at the middle of the filament (Anna et al. 1999; Marín et al. 2013). Recently, within our group, a further improved filament extensional stretching rheometer (FiSER) design was realized that allows to measure the extensional rheological properties while in situ structure characterizations can be performed (Pepe et al. 2020). Thereto, both pistons move at equal speed in opposite directions relying on the underlying control mechanism whereby the stagnation point is positioned at the center of the filament.

Besides improvements of the design and operating principles of the filament stretching rheometers, analytical models and numerical simulations have allowed to derive correction factors that can compensate for the errors introduced by the shear contribution near the plates. Using a lubrication analysis for small initial aspect ratios (ratio of sample height over diameter), Spiegelberg et al. (1996) derived a first correction factor by considering an inverse squeeze flow problem. The correction is shown to depend on the initial aspect ratio and instantaneous Hencky strain. Nielsen et al. (2008) provide an alternative notation of the same correction factor, which allows to separately insert the strains exhibited during pre-stretch and during the actual extension experiment. By performing numerical simulations for Newtonian fluids, Rasmussen et al. (2010) proposed an empirical improvement of the correction factor, which was later rewritten in terms of pre-strain and actual strain by Huang et al. (2012). Based on the principle of linear viscoelasticity, the derived correction factors for Newtonian fluids are valid for all materials, as long as they are deformed within the linear viscoelastic deformation range (Spiegelberg et al. 1996). Yao and McKinley (1998) found that for small strains, the fluid deformation in a FiSER is mainly determined by the Newtonian solvent contribution to the stress and the filament deformation is 
comparable in both the Newtonian and viscoelastic cases. However, at large strains elastic stresses dominate leading to strain-hardening in the axial mid-regions of the filament. Moreover, at larger strains the initial non-homogeneous flow, resulting from the shear regions near the endplates, leads to the growth of viscoelastic stress boundary layers near the free surface which can significantly affect the measured transient extensional viscosity (Yao and McKinley 1998). Despite the fact that a few authors performed full numerical simulations of the filament stretching process for viscoelastic fluids undergoing nonlinear deformations, the validity of the available correction factors in this regime has not been investigated. From the findings of Yao and McKinley (1998) it is expected that at very small strains the available (Newtonian) correction factors are valid. However, at moderate to large strains, the strain hardening and viscoelastic stress boundary layers can not be described by a Newtonian model and hence the correction factors are not expected to be valid. Moreover, Bach et al. (2003) state that the correction factor is less appropriate for highly non-linear materials, for which the shearing of the sample is located very close to the end plates.

The objective of this work is to investigate whether a general expression for the shear correction factor for filament stretching rheometers can be determined, which is valid in both the linear as well as in the nonlinear deformation region. By applying fully resolved numerical finite element simulations of the fluid flow in the filament stretching rheometer, the extensional flow and resulting stresses on the pistons are determined for a set of three polymers that were chosen to exhibit distinctly different rheological behaviour in shear and/or extension. An artificial material with more pronounced non-linear effects is introduced as well, to investigate the extremities of the parameter space. To describe the rheological behaviour, the eXtended Pom-Pom (XPP) constitutive model is used, since it nicely captures the physics of branched and linear polymers, as shown by Verbeeten et al. $(2001,2004)$. The solution of the XPP model for a pure uniaxial flow will be used as the ideal reference case. By using this solution in the correction factor, instead of the analytical Newtonian solution for pure uniaxial flows, it is hypothesised that even for non-linear viscoelastic polymers a material-independent correction factor can be derived. The correction factor is expected to be similar to the correction factors found by Spiegelberg et al. (1996) and Rasmussen et al. (2010) which only depend on the initial aspect ratio and the instantaneous Hencky strain.

The models used to simulate the flow in the FiSER will be explained in "Modelling". The numerical methods used to solve these models will be reviewed in "Numerical method". Subsequently in "Implementation of rheological characterization", the method to measure the shear contributions with the numerical model is described. Thereafter, in "Results and discussion", results are shown from the numerical model where first a validation of the numerical simulations is given. Finally, the results of the numerical measurements of the shear contribution and a definition for the correction factor is discussed.

\section{Modelling}

To perform simulations of the extensional flow in a FiSER, multiple models have to be used. Firstly, the initial geometry of the sample has to be determined. Subsequently, the flow equations have to be solved on this geometry using the appropriate boundary conditions. To do so, the non-linear polymers have to be modeled using a suitable constitutive equation. For this constitutive model, both linear and nonlinear material properties are needed.

\section{Geometry}

The extensional flow is created in a FiSER, by simultaneously moving two pistons in opposite directions with equal velocities. To minimize the computational cost, only a part of the FiSER is modelled, as shown in Fig. 1. Axisymmetry is assumed on curve $\Gamma_{3}$. The shape of the domain is determined by the radius of the piston $R_{\mathrm{p}}$, the mid-radius $R(t)$ and the length $L(t)$. In FiSER experiments, the sample is slightly compressed after loading to a length $L_{\mathrm{c}}$ to ensure good contact with the plates. The aspect ratio of the

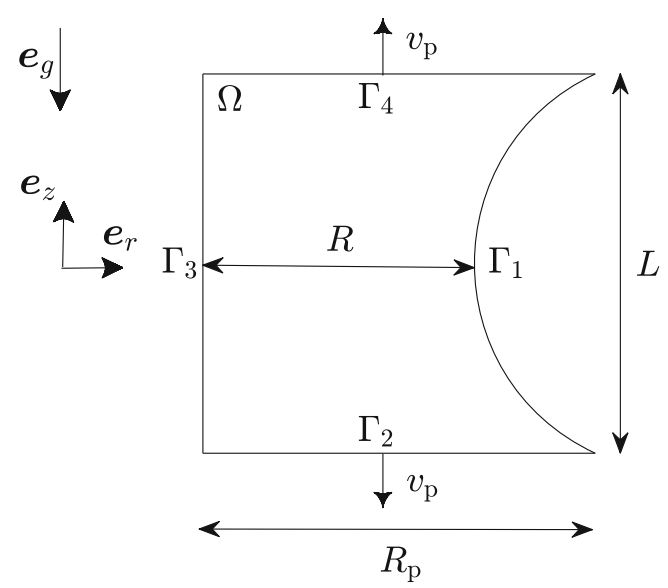

Fig. 1 Problem description of the viscoelastic extensional flow in a FiSER. The flow is set up by moving the end-plates in opposite directions with equal velocities $v_{\mathrm{p}}$. The boundaries connecting the fluid to the upper piston and the bottom piston are denoted as $\Gamma_{2}$ and $\Gamma_{4}$, respectively. The free surface is denoted by $\Gamma_{1}$ and the axisymmetric boundary is denoted as $\Gamma_{3}$ 
compressed sample is then defined as $\Lambda_{\mathrm{c}}=L_{\mathrm{c}} / R_{\mathrm{c}}$, with $R_{\mathrm{c}}$ the radius of the sample after compression. Here, it is assumed that the compressed sample is a perfect cylinder, or in other words the radius after compression $R_{\mathrm{c}}$ equals the piston radius $R_{\mathrm{p}}$ (see Table 1). In addition, a slow pre-stretch is used in experiments to increase the initial aspect ratio to $\Lambda_{0}=L_{0} / R_{0}$, where $L_{0}=L(0)$ is the length of the sample after the pre-stretch and $R_{0}=R(0)$ is the mid-radius of the sample after the pre-stretch. To reduce the computation time, the simulations start after the pre-stretch. First, the initial geometry is build, where the shape of the free surface $\Gamma_{1}$ is a circular arc or an ellipsoidal arc. An ellipsoidal arc is used when $\left(R_{\mathrm{p}}-R_{0}\right)>L_{0} / 2$ and otherwise a circular arc is used. Subsequently, the pistons are simultaneously moved apart to a length $L(t)$ (with a velocity $v_{\mathrm{p}}(t)$ ), in such a way that the middle of the sample is extended with a constant strain rate. A cylindrical coordinate system will be used throughout this paper, with components $[r, \theta, z]$. The computational domain of the FiSER is denoted by $\Omega$. Note that the negative $z$-direction is directed in the gravitation direction. Besides, a balance between surface tension, internal stresses and gravity forces determines the shape of the free surface $\Gamma_{1}$ during extension. The piston radius, the compressed radius and the mid-radius after pre-stretch are constant for all simulations done in this paper. This way, the amount of pre-strain

$\varepsilon_{\text {pre }}=2 \ln \left(R_{\mathrm{c}} / R_{0}\right)=2 \ln \left(R_{\mathrm{p}} / R_{0}\right)$

is per definition constant. Note that the logarithmic definition of the strain is used (i.e. the Hencky strain $\varepsilon$ ), because it is a consistent way of describing the strain path of a fluid element. By choosing a length after pre-strain $L_{0}$, the corresponding length after compression $L_{\mathrm{c}}$ can be determined from the initial mid-radius $R_{0}$ and plate radius
$R_{\mathrm{p}}$ using conservation of volume. So for a combination of $R_{0}$ and $R_{\mathrm{p}}$, the choice of $L_{0}$ determines the compressed aspect ratio and the initial aspect ratio. In case that $\Gamma_{1}$ is a circular arc, where $\left(R_{\mathrm{p}}-R_{0}\right)<L_{0} / 2$ applies, the analytical expression for $L_{\mathrm{c}}$ is found to be:

$$
\begin{aligned}
L_{\mathrm{c}}= & \frac{-2}{\mathrm{R}_{\mathrm{p}}^{2}}\left[\left(A \sqrt{B^{2}-A^{2}}+B^{2} \operatorname{asin}\left(\frac{A}{B}\right)\right)\right. \\
& \left.\left(B+R_{0}\right)+\frac{A^{3}}{3}-A\left(\left(B+R_{0}\right)^{2}+B^{2}\right)\right],
\end{aligned}
$$

with $\quad A=L_{0} / 2 \quad$ and $\quad B=\frac{\left(L_{0} / 2\right)^{2}+\left(R_{\mathrm{p}}-R_{0}\right)^{2}}{2\left(R_{\mathrm{p}}-R_{0}\right)}$.

For an ellipsoidal arc, where $\left(R_{\mathrm{p}}-R_{0}\right) \geq L_{0} / 2$ applies, the following analytical expression for $L_{c}$ is derived:

$$
\begin{aligned}
L_{\mathrm{c}}= & L_{0}\left[\frac{\pi}{2}\left(\exp \left(-\frac{\varepsilon_{\text {pre }}}{2}\right)-1\right)+\right. \\
& \left.\frac{1}{3}\left(2 \exp \left(-\varepsilon_{\text {pre }}\right)-4 \exp \left(-\frac{\varepsilon_{\text {pre }}}{2}\right)+5\right)\right] .
\end{aligned}
$$

Note that for a circular arc, the expression for $L_{c}$ cannot be rewritten in terms of pre-strain as for the expression of the ellipsoidal arc. Nevertheless, the circular arcs are used because they give better approximations of the real shape of the free surface at larger initial aspect ratios. In Table 1, an overview is given of the four different computational domains used in this paper. Only for the geometry with the smallest initial aspect ratio, $N_{\mathrm{g}}=4$, an ellipsoidal arc is used.

It has been verified that the ellipsoidal arc and the circular arc used in the simulated geometries in Table 1 are realistic. For these validating simulation, the pre-stretching is simulated using a velocity of $v_{\mathrm{p}}=0.03 \mathrm{~m} / \mathrm{s}$. The resulting

\begin{tabular}{|c|c|c|c|c|c|c|c|c|}
\hline$N_{\mathrm{g}}$ & $\begin{array}{l}R_{\mathrm{c}} \\
{[\mathrm{mm}]}\end{array}$ & $\begin{array}{l}L_{\mathrm{c}} \\
{[\mathrm{mm}]}\end{array}$ & $\Lambda_{\mathrm{c}}$ & $\begin{array}{l}\text { Compressed } \\
\text { shape }\end{array}$ & $\begin{array}{l}R_{0} \\
{[\mathrm{~mm}]}\end{array}$ & $\begin{array}{l}L_{0} \\
{[\mathrm{~mm}]}\end{array}$ & $\Lambda_{0}$ & $\begin{array}{l}\text { Initial } \\
\text { shape }\end{array}$ \\
\hline 1 & 1.5 & 1.5 & 1.0 & & 1.0 & 2.5 & 2.5 & \\
\hline 2 & 1.5 & 0.73 & 0.5 & & 1.0 & 1.27 & 1.27 & \\
\hline 3 & 1.5 & 0.63 & 0.42 & 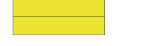 & 1.0 & 1.13 & 1.13 & \\
\hline 4 & 1.5 & 0.37 & 0.25 & 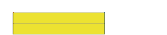 & 1.0 & 0.68 & 0.68 & \\
\hline
\end{tabular}
initial geometries show good agreement with the geometries created with circular and ellipsoidal arcs.

Table 1 Overview of the geometries studied

The dimensions of the compressed and pre-stretched state are given. Simulations start from the (pre-stretched) initial shape. A constant pre-strain $\varepsilon_{\text {pre }}=0.81$ is chosen 


\section{Flow equations}

To solve the flow in the filament stretching rheometer, the momentum and mass balance have to be solved. The following equations are used for the balance of momentum and the balance of mass (assuming an incompressible) fluid:

$$
\begin{aligned}
\rho \frac{D \boldsymbol{u}}{D t} & =-\nabla p+\nabla \cdot \boldsymbol{\tau}+\nabla \cdot\left(2 \eta_{s} \boldsymbol{D}\right)+\rho g \boldsymbol{e}_{\boldsymbol{g}} & & \text { in } \Omega, \\
\nabla \cdot \boldsymbol{u} & =0 & & \text { in } \Omega,
\end{aligned}
$$

where $\boldsymbol{u}$ is the fluid velocity, $\rho$ the density of the material, $p$ the pressure and $\boldsymbol{D}$ the deformation rate tensor. The extra stress tensor is defined by $\boldsymbol{\tau}$. A relatively small viscous component $\eta_{s}$ is added for numerical reasons and $g$ and $\boldsymbol{e}_{\boldsymbol{g}}$ are the magnitude and direction of gravity, respectively. Since the XPP constitutive equation will be used, the extra stress tensor is given by:

$\boldsymbol{\tau}=\sum_{i} g_{i}\left(\boldsymbol{c}_{i}-\boldsymbol{I}\right)$.

Here, $\boldsymbol{c}_{i}$ is the conformation tensor of mode $i$ and $g_{i}$ is the modulus of mode $i$, which equals $\eta_{i} / \lambda_{i}$ with $\eta_{i}$ the polymer viscosity of mode $i$ and $\lambda_{i}$ the polymer relaxation time of mode $i$. The constitutive model used for predicting the conformation tensor is the multi-mode XPP model (Verbeeten et al. 2004). Originally, the XPP model was proposed for branched polymers, but it also captures the physics of linear polymers, as shown by Verbeeten et al. (2001) for high-density polyethylene (HDPE). The differential equation of the XPP model is given by:

$$
\begin{aligned}
& \stackrel{\nabla}{\boldsymbol{c}_{i}}+2 \frac{\exp \left[v_{i}\left(\sqrt{\operatorname{tr} \boldsymbol{c}_{i} / 3}-1\right)\right]}{\lambda_{s, i}}\left(1-\frac{3}{\operatorname{tr} \boldsymbol{c}_{i}}\right) \boldsymbol{c}_{i} \\
& +\frac{1}{\lambda_{b, i}}\left(\frac{3 \boldsymbol{c}_{i}}{\operatorname{tr} \boldsymbol{c}_{i}}-\boldsymbol{I}\right)=\mathbf{0},
\end{aligned}
$$

where $\stackrel{\nabla}{\boldsymbol{c}_{i}}$ denotes the upper convected derivative of the conformation tensor of mode $i, \lambda_{b, i}$ denotes the relaxation time for backbone tube orientation of mode $i, \lambda_{s, i}$ denotes the backbone stretch relaxation time of mode $i$ and the parameter $v_{i}$ depends on the number of arms of the molecule $q_{i}$ following $v_{i}=2 / q_{i}$ (Verbeeten et al. 2004). Isothermal experiments are considered, wherein the temperature is constant everywhere.

\section{Boundary conditions}

The fluid is at rest at the start of the simulation $(t=0)$. The initial geometry of the filament is given in Fig. 1. On the driving pistons, the velocity is prescribed as:

- $u_{r}=0$ on $\Gamma_{2}$ and $\Gamma_{4}$,

- $u_{z}=-v_{\mathrm{p}}$ on $\Gamma_{2}$,

- $u_{z}=v_{\mathrm{p}}$ on $\Gamma_{4}$.
On the symmetry line, the following applies:

- $u_{r}=0$, on $\Gamma_{3}$.

The remaining boundary is a free surface:

- $\boldsymbol{u} \cdot \boldsymbol{n}=0$, on $\Gamma_{1}$,

where $\boldsymbol{n}$ is the outward pointing normal vector on the surface $S$. On this boundary, the surface tension is applied using a Neumann boundary condition

- $\quad\left(-p \boldsymbol{I}+2 \eta_{s} \boldsymbol{D}+\boldsymbol{\tau}\right) \cdot \boldsymbol{n}=\nabla_{s} \cdot(\hat{\gamma}(\boldsymbol{I}-\boldsymbol{n n}))$, on $\Gamma_{1}$,

with $\nabla_{s}$ the surface gradient operator. A constant surface tension, $\hat{\gamma}$ is assumed.

\section{Materials}

The first material used in this study is an isotactic polypropylene (iPP) homopolymer (Borealis HD601CF, $M_{w}=365 \mathrm{~kg} / \mathrm{mol}$, and $M_{n}=68 \mathrm{~kg} / \mathrm{mol}$ ), which is characterized and examined in other studies (Housmans et al. 2009; van Erp et al. 2013; Roozemond et al. 2015; Grosso et al. 2019). The second material that is used is a metallocene linear low-density polyethylene (LLDPE, ExxonMobil, $M_{\mathrm{w}}=94 \mathrm{~kg} / \mathrm{mol}$, and $M_{\mathrm{n}}=24 \mathrm{~kg} / \mathrm{mol}$ ), and the material is characterized in the work of van Drongelen et al. (2015). The rheology of both materials is fitted with the XPP constitutive model given in Eq. 7 . The corresponding relaxation spectra and XPP parameters are given in Tables 2 and 3. In case of the iPP, two different sets of XPP parameters are reported in literature. The first set is given by Roozemond et al. (2015). Grosso et al. (2019) modified these XPP parameters because of molecular considerations. The behaviour of both the iPPs and of the LLDPE under shear and extension is shown in Fig. 2. These materials and their characterization are chosen

Table 2 Viscoelastic model parameters of the XPP model for iPP at a reference temperature of $T=220^{\circ} \mathrm{C}$

\begin{tabular}{llllllll}
\hline & & & \multicolumn{2}{l}{ Roozemond et al. } & & \multicolumn{2}{l}{ Grosso et al. } \\
\cline { 7 - 8 }$i$ & $\eta_{i}[\mathrm{~Pa} \mathrm{~s}]$ & $\lambda_{b, i}[\mathrm{~s}]$ & $\lambda_{s, i}[\mathrm{~s}]$ & $v_{i}[-]$ & & $\lambda_{s, i}[\mathrm{~s}]$ & $v_{i}[-]$ \\
\hline 1 & 30.00 & $5 \cdot 10^{-5}$ & $2 \cdot 10^{-5}$ & 2 & & $6.0 \cdot 10^{-6}$ & 0.05 \\
2 & 130.76 & 0.0014 & $4 \cdot 10^{-4}$ & 2 & & $6.7 \cdot 10^{-6}$ & 0.05 \\
3 & 303.60 & 0.011 & 0.0027 & 2 & & $5.2 \cdot 10^{-5}$ & 0.05 \\
4 & 480.00 & 0.060 & 0.015 & 2 & & $2.9 \cdot 10^{-4}$ & 0.05 \\
5 & 377.00 & 0.29 & 0.073 & 2 & & 0.0014 & 0.05 \\
6 & 183.70 & 1.67 & 0.42 & 0.25 & & 0.0080 & 0.05 \\
7 & 46.00 & 11.5 & 2.21 & 0.17 & 0.055 & 0.02 \\
\end{tabular}

Columns 4 and 5 give the spectra used by Roozemond et al. (2015), while columns 6 and 7 provide the values derived by using molecular considerations by Grosso et al. (2019) 
Table 3 Viscoelastic model parameters of the XPP model for LLDPE (van Drongelen et al. 2015) and the arbitrary non-linear material at a reference temperature of $T=160^{\circ} \mathrm{C}$

\begin{tabular}{|c|c|c|c|c|c|c|c|}
\hline \multirow[b]{2}{*}{$i$} & \multirow[b]{2}{*}{$\eta_{i}[\mathrm{~Pa} \mathrm{~s}]$} & \multicolumn{3}{|c|}{ LLDPE Van Drongelen et al. } & \multicolumn{3}{|c|}{ Arbitrary non-linear material } \\
\hline & & $\lambda_{b, i}[\mathrm{~s}]$ & $\lambda_{s, i}[\mathrm{~s}]$ & $v_{i}[-]$ & $\lambda_{b, i}[\mathrm{~s}]$ & $\lambda_{s, i}[\mathrm{~s}]$ & $v_{i}[-]$ \\
\hline 1 & 392.42 & $7.7 \cdot 10^{-4}$ & $1 \cdot 10^{-6}$ & 2 & $2.7 \cdot 10^{-4}$ & $2 \cdot 10^{-4}$ & 2.66 \\
\hline 2 & 1196.9 & 0.00581 & $1 \cdot 10^{-6}$ & 1 & $5.8 \cdot 10^{-4}$ & $2 \cdot 10^{-4}$ & 2.66 \\
\hline 3 & 2702.0 & 0.0356 & $1 \cdot 10^{-6}$ & 0.66 & 0.00356 & $2 \cdot 10^{-4}$ & 2.66 \\
\hline 4 & 4222.2 & 0.227 & 0.0210 & 0.66 & 0.0227 & 0.21 & 2.66 \\
\hline 5 & 8522.1 & 1.53 & 0.0441 & 0.65 & 0.153 & 0.44 & 2.65 \\
\hline 6 & 13246 & 8.95 & 2.29 & 0.65 & 0.895 & 22.9 & 2.65 \\
\hline 7 & 15179 & 55.4 & 16.2 & 0.55 & 5.54 & 162 & 2.55 \\
\hline
\end{tabular}

because of the shear thinning behaviour, the non-linear extensional behaviour, the difference in viscosities between iPP and LLDPE (but similar shear viscosity of the iPPs) and the qualitative difference of the viscosity as a function of extension and shear rate.

From Fig. 2 it can be concluded that the non-linear behavior is completely different within this set of materials. The uniaxial extensional viscosity can be compared with the linear viscoelastic envelope (LVE). For the linear viscoelastic envelope, the extensional viscosity is found as

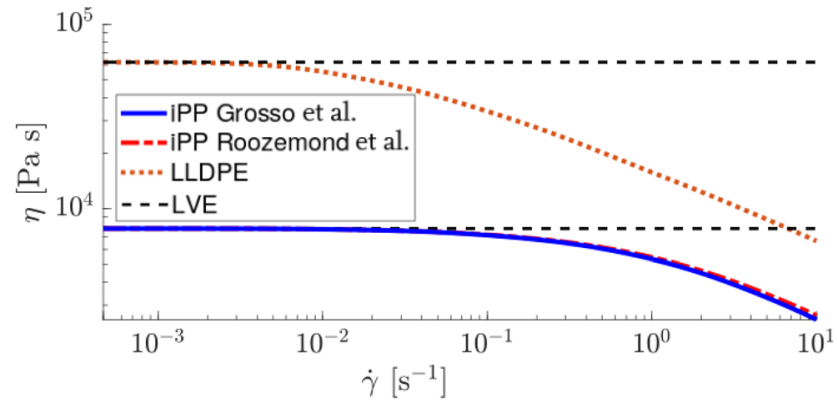

(a) Shear viscosity

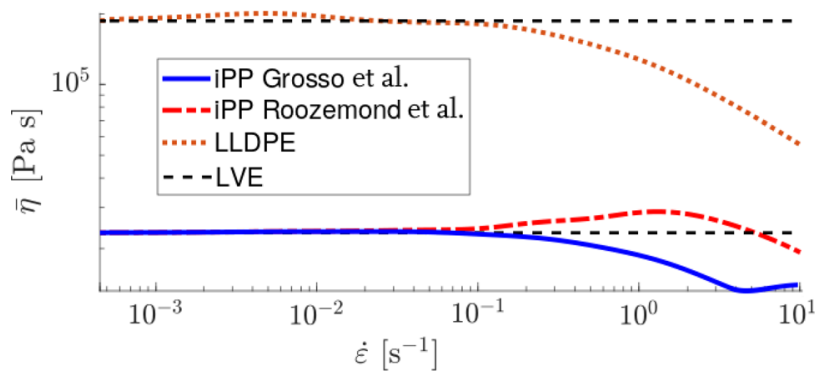

(b) Extensional viscosity

Fig. 2 Shear and extensional viscosity as a function of the shear and extension rate given by the XPP model. Three different materials are used: the $\mathrm{iPP}_{1}$ of Roozemond et al. (2015), the iPP $\mathrm{P}_{2}$ of Grosso et al. (2019) and the LLDPE of van Drongelen et al. (2015) $(T=160$ ${ }^{\circ} \mathrm{C}$ ). The linear viscoelastic prediction (LVE) for each material is also provided follows (Bird 1976; Zhang et al. 2012):

$\bar{\eta}_{\mathrm{LVE}}^{+}(t)=3\left[\eta_{s}+\sum_{i=1}^{N} \eta_{i}\left(1-\exp \left(\frac{-t}{\lambda_{b, i}}\right)\right)\right]$.

Only at very small strain rates, the non-linear XPP model equals the linear viscoelastic envelop. Therefore, it is expected that the non-linear behaviour of both materials will significantly affect the flow in the FiSER.

The rheological data at selected temperature can be superposed into a master curve at an arbitrary reference temperature by employing a temperature-dependent factor. For the rheological data given in Fig. 2, an Arrhenius-type relation is used (Morrison 2001):

$a_{T}=\exp \left[\frac{E_{a}}{R^{*}}\left(\frac{1}{T}-\frac{1}{T_{\text {ref }}}\right)\right]$,

with $a_{T}$ the temperature shift factor, $T$ the absolute temperature, $T_{\text {ref }}$ a reference temperature, $R^{*}=8.314$ $[\mathrm{J} /(\mathrm{K} \mathrm{mol})]$ the universal gas constant and $E_{a}$ the activation energy for flow. In Table 4, the activation energies and reference temperatures of the three materials are reported. Herein, also the density and surface tension are given.

\section{Numerical method}

The finite element method is used to solve the filament stretching flow problem. The initial geometry is build with Gmsh (Geuzaine and Remacle 2009). For the interpolation

Table 4 Material and rheological parameters (Roozemond et al. 2015; van Drongelen et al. 2015)

\begin{tabular}{lllll}
\hline Material & $\rho$ & $\hat{\gamma}$ & $\begin{array}{l}E_{a} \\
{[\mathrm{~kJ} / \mathrm{mol}]}\end{array}$ & $\begin{array}{l}T_{\text {ref }} \\
{[\mathrm{K}]}\end{array}$ \\
\hline iPP & 800 & 30.2 & 40.0 & 493 \\
LLDPE & {$\left[\mathrm{kg} / \mathrm{m}^{3}\right]$} & {$[\mathrm{mN} / \mathrm{m}]$} & 47.7 & 433 \\
\hline
\end{tabular}

*Also used for the arbitrary non-linear material 
of the velocity and pressure, isoparametric, triangular $P_{2} P_{1}$ (Taylor-Hood) elements are used, whereas for the conformation, triangular $P_{1}$ elements are used. In order to solve the constitutive equation (XPP), the log-conformation representation (Hulsen et al. 2005), streamline-upwind Petrov-Galerkin (SUPG) (Brooks and Hughes 1982) and DEVSS-G (Bogaerds et al. 2002) are used for stability. Below the most relevant details of the numerical procedure are outlined and more details are available in van Berlo et al. (2020).

\section{Mesh movement}

The boundary which describes the free surface will move in time and also the boundaries connected to the pistons move. Therefore, it is necessary to track these boundaries and update the mesh. The position change of the free surface is determined from the velocity at this boundary (Lagrangian based). The velocity of the free boundary is defined as:

$\frac{\mathrm{d} \boldsymbol{x}_{\Gamma_{1}}}{\mathrm{~d} t}=\boldsymbol{u}\left(\Gamma_{1}\right)$,

where $\boldsymbol{x}_{\Gamma_{1}}$ is the position of the free boundary and $\boldsymbol{u}\left(\Gamma_{1}\right)$ is the velocity at the free boundary $\Gamma_{1}$. The movement of the mesh has to be compensated. To do so, the Arbitrary Lagrangian Eulerian (ALE) formulation is used (Hirt et al. 1974). This implies that all convective terms have to be replaced with:

$\frac{D()}{D t}=\left.\frac{\partial()}{\partial t}\right|_{\zeta}+\left(\boldsymbol{u}-\boldsymbol{u}_{m}\right) \cdot \nabla()$,

where $\partial() /\left.\partial t\right|_{\zeta}$ denotes the time derivative at a fixed grid point and $\boldsymbol{u}_{m}$ is the velocity of the mesh.

\section{Weak formulation}

The weak form of the problem concerning the momentum balance and mass balance, including the boundary conditions, can be formulated as follows: find $\boldsymbol{u}, p$ and $s$ such that:

$$
\begin{array}{r}
\left((\nabla \boldsymbol{v})^{T}, \bar{v}\left(\nabla \boldsymbol{u}-\boldsymbol{G}^{T}\right)\right)+\left(\boldsymbol{D}_{v}, 2 \eta_{s} \boldsymbol{D}+\boldsymbol{\tau}\right)- \\
(\nabla \cdot \boldsymbol{v}, p)=(\boldsymbol{v}, \nabla \cdot(\hat{\gamma}(\boldsymbol{I}-\boldsymbol{n n})))_{\Gamma}+(\boldsymbol{v}, \rho \boldsymbol{b}) \\
\left(\boldsymbol{H},-\nabla \boldsymbol{u}+\boldsymbol{G}^{T}\right)=0 \\
(q, \nabla \cdot \boldsymbol{u})=0 \\
\left(\boldsymbol{d}+\bar{\tau}\left(\boldsymbol{u}-\boldsymbol{u}_{m}\right) \cdot \nabla \boldsymbol{d}, \frac{D \boldsymbol{s}}{D t}-\boldsymbol{g}(\boldsymbol{G}, \boldsymbol{s})\right)=0
\end{array}
$$

for all admissible test functions $\boldsymbol{v}, \boldsymbol{H}, q$ and $\boldsymbol{d}$. Furthermore, $\boldsymbol{D}_{v}=\left(\nabla \boldsymbol{v}+(\nabla \boldsymbol{v})^{T}\right) / 2$. In this formulation a Neumann boundary condition is used for the surface tension and gravity is introduced via the body force $\boldsymbol{b}=g \boldsymbol{e}_{\boldsymbol{g}}$. For SUPG and DEVSS-G stabilization, the parameters $\bar{\tau}$ and $\bar{\nu}$ are introduced, respectively. The conformation tensor can be derived from the log-conformation representation: $s=$ $\log c$. More information on log-conformation stabilization and the function $\boldsymbol{g}$ can be found in Hulsen et al. (2005). A projected velocity gradient $\boldsymbol{G}$ is introduced with the DEVSS-G method. Information about the discretization and this stabilization method is provided in Bogaerds et al. (2002).

\section{Time integration}

The system of equations is solved sequentially per time step. To integrate in time, a (semi-implicit) backward Euler scheme is employed for the first time step and a secondorder backward differencing scheme (semi-implicit Gear (D'Avino et al. 2012)) is employed for all subsequent time steps. The system of equations on the moving domain $\Omega$ is solved using the following steps:

First, the velocity and conformation are predicted from previous time steps using

$\hat{\boldsymbol{u}}^{n+1}=\boldsymbol{u}^{n}$

$\hat{c}^{n+1}=c^{n}$

for the first time step, and

$\hat{\boldsymbol{u}}^{n+1}=2 \boldsymbol{u}^{n}-\boldsymbol{u}^{n-1}$

$\hat{\boldsymbol{c}}^{n+1}=2 \boldsymbol{c}^{n}-\boldsymbol{c}^{n-1}$

for all subsequent time steps. Here, $\hat{\boldsymbol{u}}^{n+1}$ is the prediction of the velocity and $\hat{\boldsymbol{c}}^{n+1}$ the prediction of the conformation tensor for time $t^{n+1}$, and $\boldsymbol{u}^{n}, \boldsymbol{u}^{n-1}, \boldsymbol{c}^{n}$ and $\boldsymbol{c}^{n-1}$ are the velocities, and conformation tensors at time $t^{n}$ and $t^{n-1}$, respectively. Next, the velocity and pressure, $\boldsymbol{u}^{n+1}$ and $p^{n+1}$, are solved. The method of $\mathrm{D}$ 'Avino et al. (2012) for decoupling the momentum balance from the constitutive equation is applied, using DEVSS-G for stability (Bogaerds et al. 2002). After solving for the new velocities and pressures, the actual conformation tensor $\boldsymbol{c}^{n+1}$ is found using a second-order, semi-implicit Gear scheme with conformation predictions from Eq. 7, where the log-conformation representation and SUPG are used for stability. Subsequently, the boundary positions are updated, where the movement of the boundary is Lagrangian based according to Eq. 10, using a backward Euler scheme

$\boldsymbol{x}_{\Gamma}^{n+1}=\boldsymbol{x}_{\Gamma}^{n}+\boldsymbol{u}^{n+1}(\Gamma) \Delta t$.

The mesh velocities can now be obtained by numerically differentiating the mesh coordinates. The mesh velocity is obtained in each node using a first-order backward differencing scheme (Jaensson et al. 2015):

$\boldsymbol{u}_{m}^{n+1}=\frac{\boldsymbol{x}_{m}^{n+1}-\boldsymbol{x}_{m}^{n}}{\Delta t}$, 
where $\boldsymbol{u}_{m}^{n+1}$ is the mesh velocity at time $t^{n+1}$, and $\boldsymbol{x}_{m}^{n+1}$ and $\boldsymbol{x}_{m}^{n}$ are the mesh coordinates at time $t^{n+1}$ and $t^{n}$, respectively.

\section{Remeshing and projection}

As the mesh is deformed in time, because both pistons move apart, elements may become too deformed to yield accurate solutions. A new mesh is generated by tracking the deformation of each element and determining the change in area and the change in aspect ratio of these elements. The change in area, $f_{1}^{e}$, and aspect ratio, $f_{2}^{e}$, of each element are defined as (Jaensson et al. 2015):

$f_{1}^{e}=\left|\log \left(A^{e} / A_{0}^{e}\right)\right|$

$f_{2}^{e}=\left|\log \left(S^{e} / S_{0}^{e}\right)\right|$,

with $A^{e}$ the element area, $A_{0}^{e}$ the element area of the undeformed mesh and $S^{e}=\left(L_{\max }^{e}\right)^{2} / A_{0}^{e}$ the aspect ratio, where $L_{\max }^{e}$ is the maximum length of the sides of an element. Remeshing is invoked if either $f_{1}^{e}>0.6$ or $f_{2}^{e}>$ 0.6 , which coincides with a change in area or aspect ratio by a factor 1.8. Remeshing implies that a new mesh, covering the same domain as the old one, is generated using Gmsh (Geuzaine and Remacle 2009). To ensure accuracy of the solution, the free surface consists of at least 5 elements in the radial direction. This condition is only used in case the mid-radius becomes very small, where as a result the amount of elements drastically increases. Initially about 14 elements in the radial direction are used. After remeshing, the solution on the old mesh is projected onto the new one. The projection problem is solved to obtain the solution variables on the new mesh. The projection is done similar as was done by Jaensson et al. (2015).

\section{Implementation of rheological characterization}

The desired material function to be measured with a filament stretching rheometer is the transient extensional viscosity, $\bar{\eta}^{+}$, as a function of time and strain rate. This material function is defined as the average stress difference divided by the strain rate:

$\bar{\eta}^{+}(t, \dot{\varepsilon})=\frac{\left\langle\sigma_{z z}(t)-\sigma_{r r}(t)\right\rangle}{\dot{\varepsilon}}$,

where $\sigma$ is the total-stress tensor for a fluid undergoing homogeneous uniaxial extensional flow $\sigma_{z z}$ and $\sigma_{r r}$ are the total stress in radial and axial direction. In the simulations, the total-stress tensor follows from the extra stress tensor and pressure according to:

$\sigma=\tau-p I$.
As time increases, the transient extensional viscosity may reach a steady-state value, $\bar{\eta}$. The (steady-state) extensional viscosity is a material property of the fluid and is a function of only the strain rate (and temperature). To measure correct values for the extensional viscosity in a filament stretching rheometer, the stress is measured via the normal force on the bottom piston and a constant extension rate should be ensured at the mid-filament point. The implementations of both aspects are detailed below.

\section{Forces}

In our home-build FiSER, the force transducer is positioned at the bottom piston (Pepe et al. 2020). This means that during the simulations the force is measured at boundary $\Gamma_{2}$. The measurement of the force is done by calculating the reaction forces. The reaction force (or the internal force) can be found via the residual, once the solution of the momentum and mass balance problem is known (i.e. the displacement vector).

Since the boundary at the piston consists only of Dirichlet nodes, the total reaction force, acting on the piston, can be found by summation of the force contributions in the z-direction:

$F_{\mathrm{p}}=\sum\left(F_{\text {reac }, z}\right)_{\Gamma_{2}}$.

Here, $F_{\mathrm{p}}$ is the force acting on the piston and $F_{\text {reac, } z}$ is the $z$-component of the reaction force vector. In addition to the viscoelastic material stresses, surface tension and gravity also contribute to the measured force $F_{\mathrm{p}}$. Therefore, these contributions are subtracted when calculating the measured extensional viscosity (Tirtaatmadja and Sridhar 1993; Szabo 1997):

$\bar{\eta}_{R}^{+}=\frac{F_{\mathrm{p}}(t) /\left(\pi R^{2}(t)\right)-\hat{\gamma} / R(t)-\rho g V /\left(2 \pi R^{2}(t)\right)}{\dot{\varepsilon}}$,

with $V$ the volume of the polymer sample. The subscript $R$ indicates "real" in the measured viscosity, because the force contribution of the surface tension $\hat{\gamma}$ and gravity are subtracted. In this equation, the effect of inertial forces is neglected. For polymer melts, this inertia term can usually be neglected in the determination of the extensional viscosity (Tirtaatmadja and Sridhar 1993). This is also the case in this study, because the Reynolds number of the flow problem is much smaller than one. The extensional viscosity measured with the FiSER simulation, as given in Eq. 28, is used to determine the shear correction factor, $f_{\text {shear. In }}$ this paper, the following definition for the shear correction factor is used:

$f_{\text {shear }}=\frac{\bar{\eta}_{\mathrm{XPP}}^{+}}{\bar{\eta}_{R}^{+}}$. 
Here, $\bar{\eta}_{\mathrm{XPP}}^{+}$is determined by solving the XPP constitutive equation (see Eq. 7) for a pure uniaxial extensional flow starting from rest. This definition is different from other work in literature about the shear correction factor. Therein, the pure uniaxial extensional viscosity was calculated using (analytical) solutions of a linear viscous model (Spiegelberg et al. 1996; Rasmussen et al. 2010). This modified definition is needed to study the validity of using a shear correction factor under non-linear conditions.

\section{Controller}

In uniaxial extension, the mid-radius of the sample has to decrease exponentially in time:

$R=R_{0} \exp \left(-\frac{1}{2} \varepsilon\right)$

with $\varepsilon$ the mid-radius-based Hencky strain. In case of a perfectly cylindrical sample, the pistons should be moved apart so that the gap between the pistons increases exponentially in time. In reality, however, the lack of uniformity of the flow along the axis of extension does not allow to a priori determine the required piston movement. Therefore, a strain-based controller is developed by Marín et al. (2013), which adjusts the piston velocity so that the mid-radius of the sample decreases exponentially in time. In the proposed scheme, the motion of the pistons is commanded by controlling their velocity via the Hencky strain. In the feed-back loop, the strain is corrected by comparing the actual radius with the ideal radius of the middle of the filament, $R_{\text {ideal }}$. To do so, the radius of the middle of the filament is measured with a laser and is used as an input in the next time step. In the simulations, the minimum radius of the free surface is determined per time step $i$ and is defined as the measured radius $R(i)$. The equation for the controller of the length-based Hencky strain is given by Marín et al. (2013):

$$
\begin{aligned}
\varepsilon_{z}(i+1)= & \varepsilon_{z}(i)+K_{p}[\delta \varepsilon(i)-\delta \varepsilon(i-1)] \\
& +K_{i} \Delta t[\delta \varepsilon(i)]+\Delta \varepsilon_{z}^{f f}(i) .
\end{aligned}
$$

The corresponding filament length and piston velocity can be calculated with the following relations:

$$
\begin{aligned}
L(i+1) & =L_{0} \exp \left(\varepsilon_{z}(i+1)\right) . \\
v_{\mathrm{p}}(i+1) & =\frac{L(i+1)-L(i)}{\Delta t} .
\end{aligned}
$$

In control language, $\varepsilon_{z}$ is the actuated variable and $\varepsilon$ the controlled variable. In Eq. 31, the error $\delta \varepsilon$ is calculated as follows:

$\delta \varepsilon(i)=\varepsilon_{\text {ideal }}(i)-\varepsilon(i)=2 \ln \left(\frac{R(i)}{R_{\text {ideal }}(i)}\right)$

where $\varepsilon(i)$ is the measured mid-radius-based Hencky strain at time step $i$, which follows from the measured radius using
Eq. 30. $R_{\text {ideal }}$ is the ideal radius for pure uniaxial flow. The ideal radius also follows from Eq. 30 using that $\varepsilon(t)=\dot{\varepsilon} t$. The actual radius of the sample is measured with a laser and is defined as $R$ ( $=R_{\text {meas }}$ ). In the home-build FiSER, the time step of the laser is $\Delta t=200 \mu \mathrm{s}$ and the PI gains are $K_{p}=0$ and $K_{i}=2.5 \mathrm{~s}^{-1}$ (Pepe et al. 2020). However, in the simulations $K_{i} \Delta t=0.08$ is used. At the largest strain rate of $10 \mathrm{~s}^{-1}$, a time step of $20 \mu \mathrm{s}$ is used and hence $K_{i}=4000 \mathrm{~s}^{-1}\left(K_{p}=0\right)$. The time step and integral gain depends linearly on the strain rate, with lower limits of $\Delta t=200 \mu \mathrm{s}$ and $K_{i}=400 \mathrm{~s}^{-1}$, respectively. For the controller and the simulation, the same time step is used. Also a feed-forward contribution $\Delta \varepsilon_{z}^{f f}$ is present in the controller of the home-build FiSER. However, in the simulations, this feed-forward contribution is superfluous and therefore set to zero $\left(\Delta \varepsilon_{z}^{f f}=0\right)$.

\section{Results and discussion}

First, a validation of the simulations is given. Here, the controlled radius is validated and the simulated forces are compared with measurements from literature. This is followed by a convergence study. Subsequently, strain rate distributions over the radius of the sample are shown. Finally, the shear correction factor is discussed, where the effects of initial aspect ratio and strain rate on the shear correction factor are shown.

\section{Validation}

One of the requirements for a pure uniaxial flow is that the mid-radius of the sample decreases exponentially with strain. As mentioned before, a controller is used to ensure this exponential decrease (see "Controller"). In Fig. 3, the mid-radius of the filament is given as a function of the Hencky strain. Here, the simulated radius immediately (after

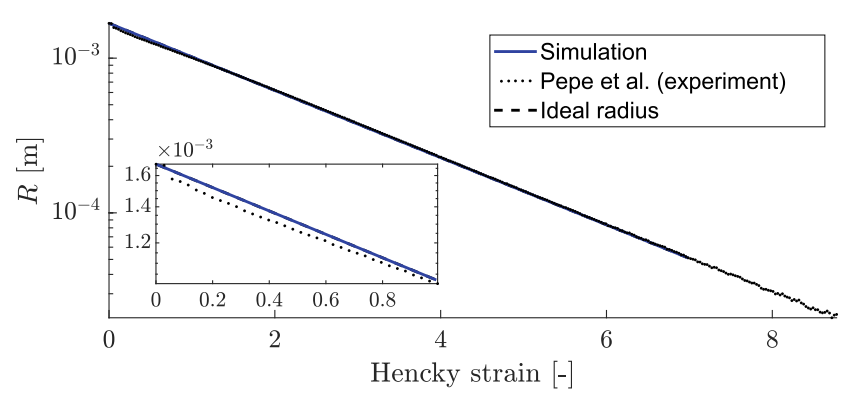

Fig. 3 Mid-radius measurements with the home-build FiSER at a strain rate of $\dot{\varepsilon}=0.71 \mathrm{~s}^{-1}$ and temperature of $150{ }^{\circ} \mathrm{C}$ (Pepe et al. 2020). The sample dimensions are: $R_{\mathrm{c}}=4 \mathrm{~mm}, L_{\mathrm{c}}=1.14 \mathrm{~mm}$, $R_{0}=1.68 \mathrm{~mm}$ and $L_{0}=3.64 \mathrm{~mm}$. The solid line represents the simulation of the FiSER with the XPP model and the dashed line is the ideal mid-radius following from Eq. 30 
one time step of $\varepsilon=0.015)$ follows the ideal exponential profile. The slopes of the measured and ideal radius are identical. This means that the strain-based controller of the simulations is fast and works properly. In the FiSER experiments, the controller is slower, because a lower value of the integral gain $\left(K_{i}=2.5 \mathrm{~s}^{-1}\right)$ is used.

To validate the simulations, the force results are compared with simulated force results from the work of Kolte et al. (1997). Herein, the flow of polyisobutylene (PIB) in a FiSER is simulated using a multi-mode viscoelastic Oldroyd-B constitutive equation. Tirtaatmadja and Sridhar (1993) performed FiSER measurements with an exponential decreasing mid-radius and measured the force at the plate. A comparison of all simulated and experimentally determined forces is given in Fig. 4. For the setup used by Tirtaatmadja and Sridhar (1993) the early response of the fluids is not only masked by the shear correction factors but also by the dynamics of the drive train. The drive has to accelerate from zero velocity to a large velocity instantaneously $\left(L_{0} \dot{\varepsilon}\right)$, which takes approximately 0.1 seconds in this experiment. Note that for more recent filament stretching rheometers, drives accelerate faster and controllers can follow the desired exponential radius evolution from very small strains (see Fig. 3). Hence, the effects of acceleration are not studied in this paper. In Fig. 4, it can also be seen that our simulated force matches the simulated force of Kolte et al. (1997). For the used OldroydB model an analytical solution for the purely uniaxial extensional viscosity can be found as (Kolte et al. 1997):

$$
\begin{aligned}
\bar{\eta}_{\mathrm{Odd}-\mathrm{B}}^{+}(t, \dot{\varepsilon})= & 3 \eta_{s}+\sum_{i=1}^{N} \eta_{i}\left(\frac{3}{\left(1-2 \mathrm{De}_{i}\right)\left(1+\mathrm{De}_{i}\right)}\right. \\
& \left.-\frac{2 \exp \left[-\left(\frac{1}{\mathrm{De}_{i}}-2\right) \dot{\varepsilon} t\right]}{1-2 \mathrm{De}_{i}}-\frac{\exp \left[-\left(\frac{1}{\mathrm{De}_{i}}+1\right) \dot{\varepsilon} t\right]}{1+\mathrm{De}_{i}}\right),
\end{aligned}
$$

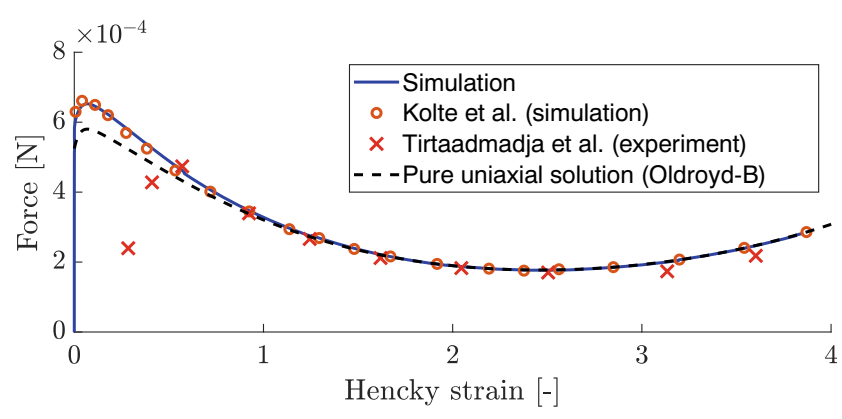

Fig. 4 Force as a function of the Hencky strain of a sample extended with a strain rate of $\dot{\varepsilon}=2.0 \mathrm{~s}^{-1}$ using a four mode Oldroyd-B model. The solvent viscosity, polymer viscosities and relaxation times of the PIB used are $\eta_{s}=12.4 \mathrm{~Pa} \mathrm{~s}, \eta=\left[\begin{array}{llll}1.69 & 2.56 & 2.53 & 1.85\end{array}\right] \mathrm{Pa} \mathrm{s}$ and $\lambda=\left[\begin{array}{lllll}4.20 & 1.12 & 0.167 & 0.0149\end{array}\right] \mathrm{s}$. The surface tension is neglected and the sample dimensions are $L_{\mathrm{c}}=L_{0}=1.5 \mathrm{~mm}$ and $R_{\mathrm{c}}=R_{0}=1.5$ $\mathrm{mm}$ with $\mathrm{De}_{i}=\dot{\varepsilon} \lambda_{i}$ the Deborah number for the $i$ 'th mode and $N$ the number of modes. With this extensional viscosity, the analytical (pure uniaxial) force is found by using Eq. 28, whereby that gravity and surface tension are not contributing. This pure uniaxial solution is also shown in Fig. 4. By comparing the simulations and the pure uniaxial solution, it can be concluded that at the start of the simulations, the flow is not purely uniaxial, since the force overshoot is larger in the simulation of an actual filament stretching experiment. This difference between the pure uniaxial force and the experimental force is a result of shear contributions near the plates (Spiegelberg et al. 1996). To simulate the flow in the FiSER with the XPP model, the same governing equations as for the OldroydB model are used. Therefore, only the constitutive equation is changed when using the XPP model. In the work of Baltussen et al. (2010) it can be seen that the XPP model is correctly implemented in the in-house developed software package TFEM.

\section{Mesh and time step convergence}

To study the mesh convergence of the system, simulations are performed on five different meshes given in Table 5 . Here, $n_{\text {ini }}=R_{\mathrm{p}} / h_{\text {ini }}$ is the number of elements at the piston boundaries, with $h_{\text {ini }}$ the initial element size. At the start of the experiment, the initial element size is used in the entire domain. For the convergence study, remeshing is included. During remeshing, the mesh at the middle of the filament is refined, while the amount of elements at the boundary is kept the same as the initial element size. Remeshing is performed such that at least $n_{\text {mid }}=5$ elements are present over the mid-radius $R$ ( $h_{\text {mid }} \leq R / n_{\text {mid }}$ ), even at large strains where the radius becomes very small. Simulations up to a strain of $\varepsilon=1.5$ are performed, with a time step of $\Delta t=$ $10^{-5} \mathrm{~s}$, a strain rate of $\dot{\varepsilon}=10 \mathrm{~s}^{-1}$ and a temperature of $T_{0}=200{ }^{\circ} \mathrm{C}$. The sample dimensions are $R_{\mathrm{p}}=4, R_{0}=3$ and $L_{0}=6 \mathrm{~mm}$. In Fig. 5, the simulated forces are shown for the different meshes. At a strain of 1.5, the simulated force of the reference solution is close to the pure uniaxial force determined with the XPP model (as expected). Also a

Table 5 Mesh resolution of different surface meshes in the convergence study

\begin{tabular}{llll}
\hline Mesh & $n_{\text {ini }}$ & $h_{\text {ini }}[\mathrm{mm}]$ & $n_{\text {mid }}$ \\
\hline M1 & 2 & 2.0 & 5 \\
M1 & 4 & 1.0 & 5 \\
M3 & 6 & 0.67 & 5 \\
M4 & 10 & 0.4 & 5 \\
M5* & 18 & 0.22 & 5 \\
\hline
\end{tabular}

The star symbol * indicates the reference mesh 


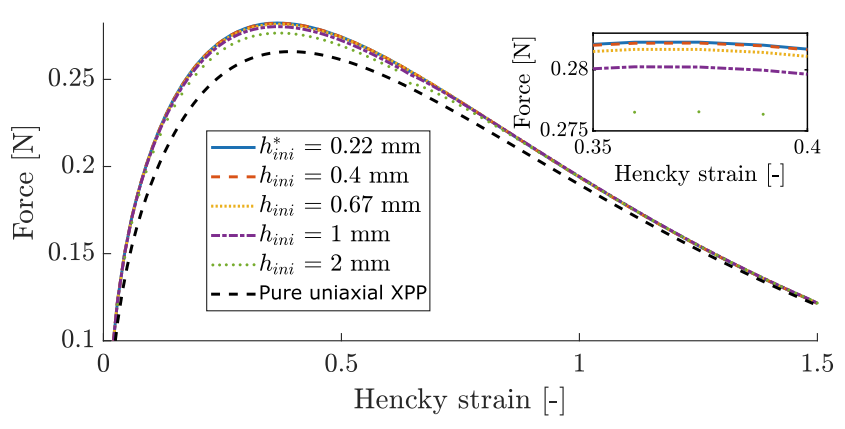

Fig. 5 Simulated forces and the pure uniaxial solution calculated with the XPP model. The reference force curve has an initial element size of $h_{\text {ini }}^{*}=0.22 \mathrm{~mm}$

convergence with increasing mesh resolution of these forces can be seen at the maximum force $(\varepsilon=0.37)$.

In order to investigate this convergence in a more quantitative manner, the following error is defined:

$\epsilon_{F}(\varepsilon)=\frac{\left|F(\varepsilon)-F^{*}(\varepsilon)\right|}{F^{*}(\varepsilon)}$,

with $F$ the simulated force and $F^{*}$ the simulated force for the reference mesh $5^{*}$ as indicated in Table 5. The error is calculated for the different meshes at a strain of $\varepsilon=0.37$ and $\varepsilon=1.5$, as shown in Fig. 6a. The convergence at a strain of 0.37 seems to be cubic. This rate of convergence is expected based on the order of interpolation of the elements (quadratic elements). For a strain of 1.5, the convergence seems to be quadratic. This is lower than the expected convergence rate. One of the possible reasons is that remeshing changes the convergence rate. Also other numerical errors can affect the convergence rate. In this work, the M4 mesh is used to simulate the flow in the FiSER. The error of this mesh compared to the reference mesh is smaller than $10^{-4}$ at a strain of 1.5.

A suitable time step has to be chosen for the simulations. Therefore, a time dependency study is performed on the M4 mesh. The same conditions are used as for the mesh dependency study. There is a limit on how large the time step size can be, because of the controller $\left(\Delta t_{\max }=2\right.$. $\left.10^{-4}\right)$. Besides, at higher strain rates, the time step is linearly decreased to maintain stability of the simulation. Equal time steps for the controller and the simulation are used. The time dependency is studied in a quantitative manner by using Eq. 36. The reference time step is taken as $\Delta t_{1}^{*}=1 \cdot 10^{-7} \mathrm{~s}$. In Fig. $6 \mathrm{~b}$, the result of the convergence with the time step is shown. For both strains, the convergence seems to be linear. This figure also shows that the errors are quite small compared to the error in the mesh convergence, indicating that it is only important that the time step is smaller than the time step limit due to the controller. The convergence rate is lower than the expected one, since a second-order time stepping scheme is used.
This is possibly caused by the remeshing and the first-order mesh movement.

\section{Shear correction factor}

In this section, FiSER simulations are investigated for multiple geometries and materials. The simulation domains used in this study are given in Table 1 . For geometry 2 (using $\Lambda_{c}=0.5$ ) at a strain rate $\dot{\varepsilon}$ of $10 \mathrm{~s}^{-1}$ the simulation results at different Hencky strains are given in Fig. 7. Herein, the shape evolution of three polymer melts (iPP (Roozemond et al. 2015), iPP 2 (Grosso et al. 2019) and LLDPE (van Drongelen et al. 2015)) and of an arbitrary non-linear material are shown. From Fig. 7 it is clear that at small strains $(\varepsilon \leq 1)$, the shapes of the free surface of the polymer melts match. But for Hencky strains larger than 1, the controller changes the velocity of the pistons to control the mid-radius. Therefore, the length of the samples and their free surface shapes are different, but the mid-radius is exactly the same.

In Fig. 8, the extensional viscosity which follows from these FiSER simulations is shown. By comparing the simulations with the pure uniaxial solutions it can be concluded that, although the strain rate in the middle of the sample is constant, the flow is not purely uniaxial at

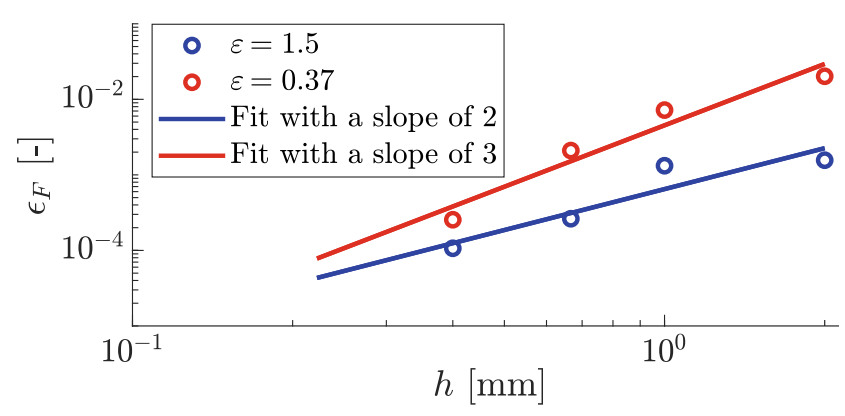

(a) Mesh size convergence

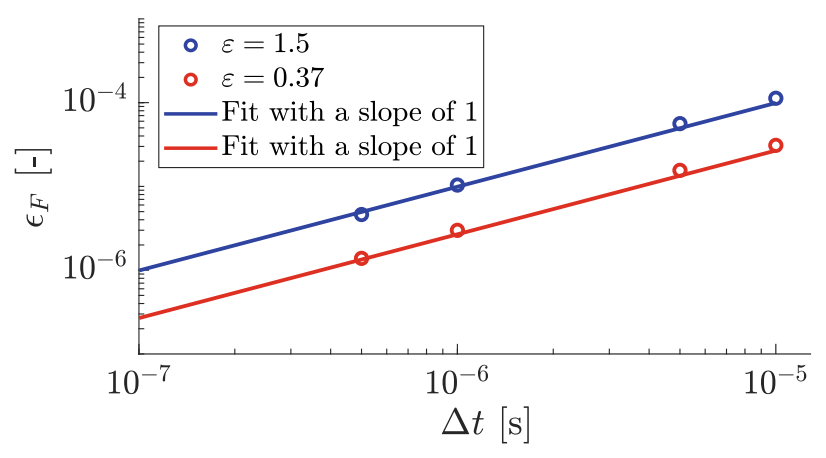

(b) Time step convergence

Fig. 6 Mesh and time step convergence at a strain of 0.37 and 1.5. In (a), a time step of $\Delta t=10^{-5} \mathrm{~s}$, a strain rate of $\dot{\varepsilon}=10 \mathrm{~s}^{-1}$ and a temperature of $T_{0}=200{ }^{\circ} \mathrm{C}$ are used. In (b), a M4 mesh is used with $\dot{\varepsilon}=10 \mathrm{~s}^{-1}$ and $T_{0}=200^{\circ} \mathrm{C}$ 


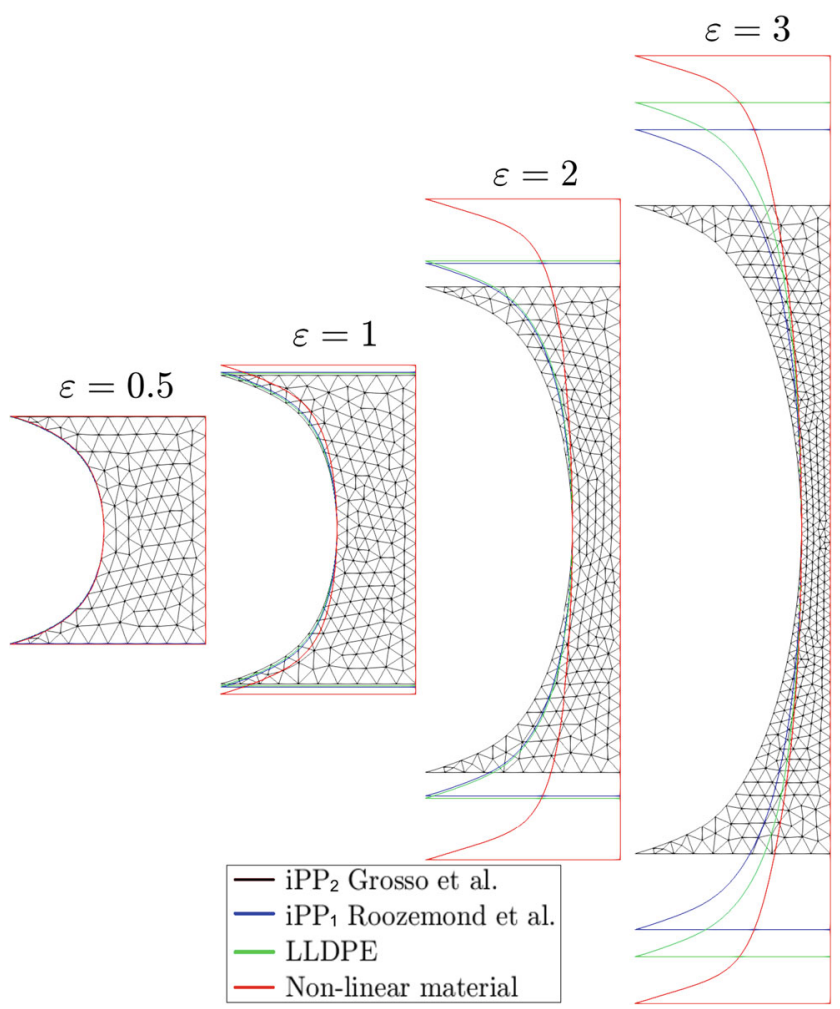

Fig. 7 Simulated boundaries of the iPPs, LLDPE and the arbitrary non-linear material at strains of $\varepsilon=0.5, \varepsilon=1, \varepsilon=2$ and $\varepsilon=3$. The second initial geometry is used with $\Lambda_{\mathrm{c}}=0.5$. The mesh evolution is shown for iPP $\mathrm{P}_{2}$ of Grosso et al. (2019) and the filaments are extended with a strain rate of $\dot{\varepsilon}=10 \mathrm{~s}^{-1}$ at $T=160{ }^{\circ} \mathrm{C}$

small strains. Therefore, the real extensional viscosity of the simulation needs to be corrected (as explained in "Forces"). At large strains $(\varepsilon>2)$, the simulations converge towards the pure uniaxial solution. This implies that there is no correction needed at large strains. In this case, the flow at the middle of the filament behaves perfectly uniaxial (like a perfect cylinder). The deviation from a pure uniaxial flow has already been shown in multiple studies (Spiegelberg et al. 1996; Nielsen et al. 2008; Rasmussen et al. 2010;
Huang et al. 2012). In the work of Spiegelberg et al. (1996) it is assumed that the fluid sample is viscous and has an initial aspect ratio smaller than one. Therefore, a lubrication approach can be used to describe the initial deformation of the fluid sample. Since this fluid sample is confined between two cylinders, the problem resembles a squeeze flow problem (with reversed direction of motion). Based on this theory, an analytical expression for the correction factor has been derived. Nielsen et al. (2008) have rewritten this analytical expression in terms of strain and pre-strain. The analytical correction factor which follows from this is (Spiegelberg et al. 1996; Nielsen et al. 2008):

$f_{\text {shear }}=\left[1+\frac{\exp \left(-7\left(\varepsilon(t)+\varepsilon_{\text {pre }}\right) / 3\right)}{3 \Lambda_{\mathrm{c}}^{2}}\right]^{-1}$.

Here, $t=0$ is defined after pre-stretch and therefore $R_{0}$ is the mid-radius of the polymer sample after pre-stretch (same definition for $\varepsilon(t)$ and $R_{0}$ as in this paper) and $R_{\mathrm{c}}$ and $\Lambda_{\mathrm{c}}$ are the compressed radius (i.e. the plate radius) and compressed aspect ratio, respectively.

Rasmussen et al. (2010) have tried to improve the analytical correction factor. They found an empirical function for the correction factor, which ensures less than $3 \%$ deviation from their FiSER simulations. A Newtonian sample was used in these simulations and the shear correction factor was determined in the linear regime. No pre-stretching was done, meaning that the simulations start with cylindrical initial geometries. These geometries have a compressed aspect ratio range of $\Lambda_{\mathrm{c}}=0.2$ to 1.5. This correction factor is rewritten in terms of strain and pre-strain by Huang et al. (2012). The empirical correction factor is (Rasmussen et al. 2010; Huang et al. 2012):

$f_{\text {shear }}=\left[1+\frac{\exp \left(-5\left(\varepsilon(t)+\varepsilon_{\text {pre }}\right) / 3-\Lambda_{\mathrm{c}}^{3}\right)}{3 \Lambda_{\mathrm{c}}^{2}}\right]^{-1}$.

The correction factors from literature do not include the strain rate. This is because the theory of Spiegelberg et al. (1996) is based on a Newtonian (viscous) fluid. In this

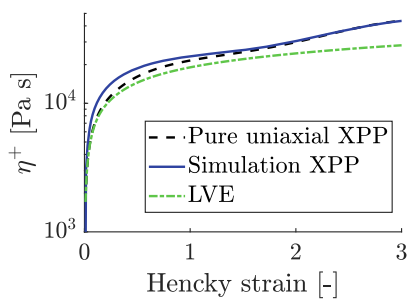

(a) LLDPE

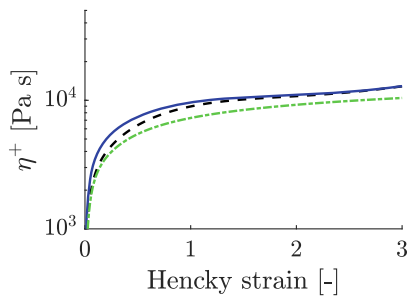

(b) iPP 1 (Roozemond et al., 2015)

Fig. 8 Extensional viscosity of a filament extended at a strain rate of $\dot{\varepsilon}=10 \mathrm{~s}^{-1}$ and a temperature of $T=160{ }^{\circ} \mathrm{C}$. Geometry 2 is used with a compressed aspect ratio of $\Lambda_{\mathrm{c}}=0.5$ (see Table 1). The numerical simulations are performed with the XPP model and the extensional

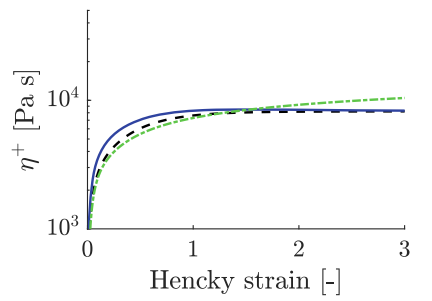

(c) iPP 2 (Grosso et al., 2019)

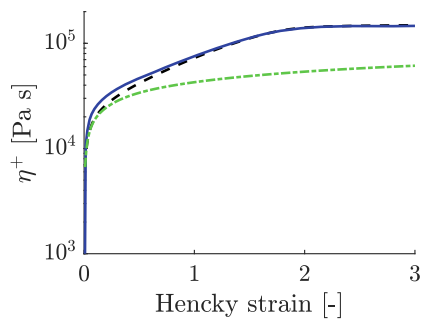

(d) Arbitrary non-linear material

viscosity is found with Eq. 28. The pure uniaxial solution is found by solving the XPP model for a pure uniaxial flow. The linear viscoelastic envelope is calculated using Eq. 8. In all figures, a similar coloring scheme is used for the various results 
type of fluids, the viscous stresses are linearly correlated to the local strain rates and therefore drop out of the analytical expression of the shear correction factor. Hence, these correction factors are only valid for small strains in the linear viscoelastic region (Spiegelberg et al. 1996). Since FiSER experiments can be in the non-linear regime even at small strains (as shown in Fig. 8), the correction factors found for Newtonian fluids are revisited in the following sections.

\section{Geometry dependency}

The non-linear shear correction factor is defined as the ratio of the pure uniaxial viscosity (XPP) and the uniaxial viscosity obtained from the FiSER simulation (as given in Eq. 29). For both iPPs and LLDPE, a clear non-linear behaviour is seen, since the simulated extensional viscosity already deviates from the LVE at very small strains (see Fig. 8a-c). The three materials show distinct differences in rheological behaviour. In Fig. 8d, the extensional viscosity of an arbitrary material, which will be indicated as nonlinear material, is presented. The extensional viscosity of this material is tuned so that it shows more pronounced non-linear behaviour at Hencky strains smaller than one as compared to the three polymer melts.

The effect of the initial aspect ratio on the correction factor is shown in Fig. 9. In this figure, it can be seen that the correction factor deviates more from 1 with a decreasing initial aspect ratio. This corresponds with the theory of Spiegelberg, where it is stated that a larger correction for shear contributions is needed for small initial aspect ratios (Spiegelberg et al. 1996). To evaluate the models given in literature, Eqs. 37 and 38 are calculated for the given initial aspect ratios. From Fig. 9, it follows that the empirical relation of Rasmussen et al. (2010) is better in predicting the correction factor than the model of Spiegelberg et al. (1996). These correction factors from literature are based on a viscous (linear) Newtonian material. But the iPP used in this study is non-linear viscoelastic. It is thus quite surprising that the empirical shear correction factor of Rasmussen et al. (2010) is quite accurate for the chosen initial aspect ratios. Also note that a different reference is used in the shear correction factor compared to the work of Rasmussen et al. (2010), see Eq. 29. It is found that the pure uniaxial solution of the non-linear constitutive model $\bar{\eta}_{\text {XPP }}^{+}$should be used instead of the linear viscoelastic viscosity $\bar{\eta}_{\mathrm{LVE}}^{+}$, because they do not match (see Fig. 8).

To define a more accurate correction factor based on the simulated (non-linear) shear correction factor in Fig. 9, a modification of the shear correction factors of Spiegelberg et al. (1996) and Rasmussen et al. (2010) is proposed. This equation is found by fitting the simulated correction factors given in Fig. 9 with the following function:

$$
f_{\text {shear }}=\left[1+\frac{\exp \left(-a\left(\varepsilon(t)+\varepsilon_{\text {pre }}\right) / 3-b\right)}{3 \Lambda_{\mathrm{c}}^{2}}\right]^{-1},
$$

with $a$ and $b$ the fitting parameters. For the four compressed aspect ratios, $a$ turns out to be approximately 4 , while $b$ decreased with increasing compressed aspect ratio. To fit $b$ per compressed aspect ratio, a function needs to be used that decreases to zero for infinite compressed aspect ratios. This because then the shape approaches a perfect cylinder for which no correction is needed. Therefore, the following exponential function is used to fit $b$ :

$b=\exp \left(-\Lambda_{\mathrm{c}} / c\right)$.

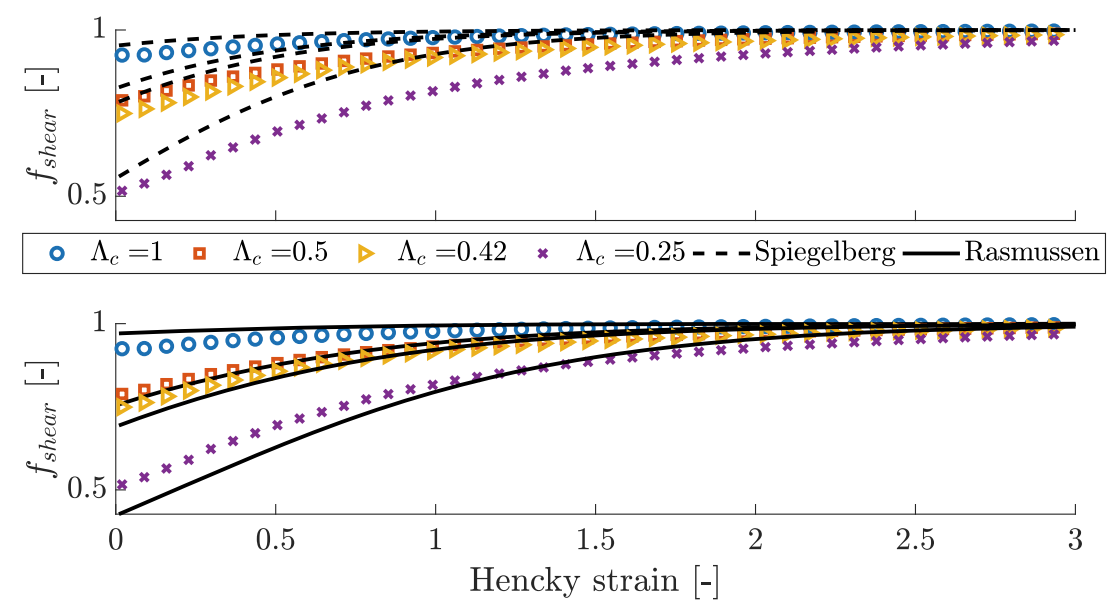

Fig. 9 Simulated shear correction factor compared with the shear correction factors found in literature for different initial aspect ratios. The top figure shows the simulation results compared to the theoretical relation of Spiegelberg et al. (1996) and (Nielsen et al. 2008) given in Eq. 37. The bottom figure shows the simulation results compared to the empirical relation of Rasmussen et al. (2010) and (Huang et al. 2012) given in Eq. 38. The iPP 2 (characterized by Grosso et al. (2019)) is extended with a strain rate of $\dot{\varepsilon}=10 \mathrm{~s}^{-1}$ at a temperature of $T=160$ ${ }^{\circ} \mathrm{C}$ 
Good agreement with $b$ per compressed aspect ratio is found for $c=1$. Combining Eqs. 39 and 40 with $a=4$ and $c=1$ results in the following proposed non-linear shear correction factor:

$f_{\text {shear }}=\left[1+\frac{\exp \left(-4\left(\varepsilon(t)+\varepsilon_{\mathrm{pre}}\right) / 3-\exp \left(-\Lambda_{\mathrm{c}}\right)\right)}{3 \Lambda_{\mathrm{c}}^{2}}\right]^{-1}$.

In Fig. 10, this proposed shear correction factor is compared with the simulated shear correction factors. It can be seen that the error of the improved correction factor relation is within $3 \%$ for all investigated compressed aspect ratios. Because of the choice of using pre-defined free surface shapes (circular and ellipsoidal), it is possible to rewrite Eq. 41 in terms of length $L_{0}$ and radius $R_{0}$ after pre-strain using Eqs. 2 and 3.

Because the proposed shear correction factor in Eq. 41 does not depend on the constitutive material behaviour, this correction can be done without a-priori knowledge on the rheological material behaviour. The measured viscosity should be corrected with the shear correction factor given in Eq. 41, i.e.:

$\bar{\eta}_{\text {corr }}^{+}=\bar{\eta}_{R}^{+} f_{\text {shear }}$,

where $\bar{\eta}_{\text {corr }}^{+}$is the corrected extensional viscosity and $\bar{\eta}_{R}^{+}$the measured viscosity which is found from the measured force on the plate and the mid-radius evolution using Eq. 28.

\section{Rate independency}

It is known that the non-linear behaviour of polymers is rate dependent. Therefore, the rate dependency of the shear correction factor is investigated. In the FiSER, the radius of the sample is controlled in order to produce a locally purely uniaxial flow. Hence, the strain as a function of time is found from $\varepsilon(t)=\dot{\varepsilon} t$. The modified shear correction factor in Eq. 41 thus only depends on the strain rate via the strain. Hence, when plotting the shear correction factor versus strain, no difference between shear correction factors at different strain rates is expected, because no other strain

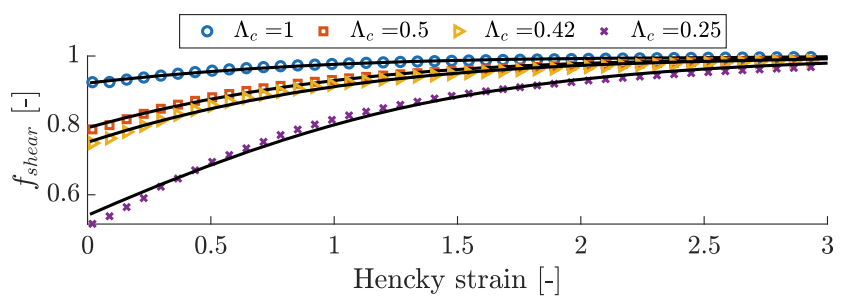

Fig. 10 Simulated correction factor compared with the modified relation for the shear correction factor given in Eq. 41 for different compressed aspect ratios. The $\mathrm{iPP}_{2}$ (characterized by Grosso et al. (2019)) is extended with a strain rate of $\dot{\varepsilon}=10 \mathrm{~s}^{-1}$ at a temperature of $T=160{ }^{\circ} \mathrm{C}$ rate dependency is present in the modified shear correction factor. In the simulations, the minimum applied strain rate is chosen as $\dot{\varepsilon}=0.71 \mathrm{~s}^{-1}$. This is a typical strain rate applied in filament stretching rheometers (Pepe et al. 2020). Anna et al. (2001) presented a detailed discussion of gravitational sagging. Herein, they show that sagging becomes significant when capillary forces in the neck near the axial mid-plane are no longer able to overcome the axial body force. This leads to a critical strain rate $\dot{\varepsilon}_{\text {sag }} \approx$ $\rho g L_{0} / \eta_{0}$, which must be exceeded in order to minimize the role of gravitational sagging. For the materials and geometries used in this study, the critical strain rate is on the order of 0.001 to $0.01 \mathrm{~s}^{-1}$. Therefore gravitational sagging plays no role in the following simulation results. The maximum applied strain rate in the FiSER simulations is $10 \mathrm{~s}^{-1}$. This is done to investigate at least one decade of strain rates. Again, geometry 2 is used with a compressed aspect ratio of $\Lambda_{\mathrm{c}}=0.5$. Only the results of $\mathrm{iPP}_{2}$ (Grosso et al. 2019) are presented here, but it should be noted that the same conclusions about the rate dependency apply to the other compressed aspect ratios and the other two polymer melts. The simulated shear correction factors at different strain rates for $\mathrm{iPP}_{2}$ are shown in Fig. 11. At a compressed aspect ratio of $\Lambda_{\mathrm{c}}=0.5$, the strain rate only slightly $(\approx 2 \%)$ affects the shear correction factors. Therefore, it is concluded that the correction factor is strain rate independent for the three polymer melts tested so far. Simulations in a range of compressed aspect ratios and strain rates are performed to verify the strain rate independency.

\section{Material independency}

According to the correspondence principle of linear viscoelasticity, the same shear correction factor should be valid for all types of fluids (Spiegelberg et al. 1996). However, in case of non-linear viscoelastic materials, this is not necessarily true, because initial flow conditions affect chain conformation at all later times (Spiegelberg et al. 1996). Therefore, the shear correction factors of the $\mathrm{iPP}_{1}$ of Roozemond et al. (2015) and the LLDPE are shown in Fig. 12. From this figure, it follows that these materials have approximately the same shear correction

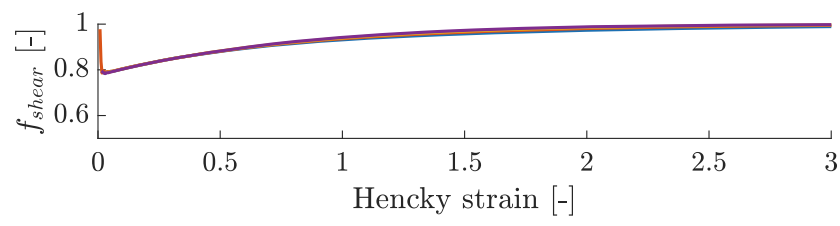

Fig. 11 Simulated correction factors for the $\mathrm{iPP}_{2}$ of Grosso et al. (2019) at strain rates of $\dot{\varepsilon}=0.71 \mathrm{~s}^{-1}, \dot{\varepsilon}=1.71 \mathrm{~s}^{-1}, \dot{\varepsilon}=4.14 \mathrm{~s}^{-1}$ and $\dot{\varepsilon}=10.0 \mathrm{~s}^{-1}$ using geometry 2 with $\Lambda_{\mathrm{c}}=0.5$ (for $T=160{ }^{\circ} \mathrm{C}$ ) 

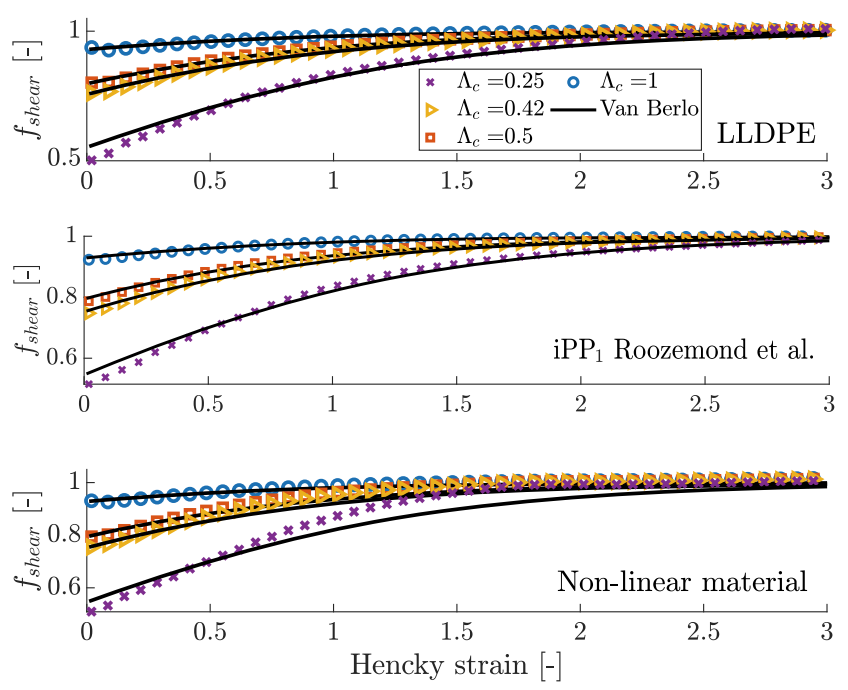

Fig. 12 Simulated correction factors for LLDPE, iPP $\mathrm{P}_{1}$ and the nonlinear material compared to the modified shear correction factor given in Eq. 41. The filaments are extended with a strain rate of $\dot{\varepsilon}=10 \mathrm{~s}^{-1}$ at a temperature of $T=160{ }^{\circ} \mathrm{C}$

factor as the $\mathrm{iPP}_{2}$ of Grosso et al. (2019). This means that although the materials exhibit completely different nonlinear rheological behaviour, the shear correction factors match. This supports the proposed shear correction factor, Eq. 41, which appears to be independent of the type of polymer and independent of the strain rate.

Although the polymers have distinctive rheological behaviours, it follows from Fig. 7 that for a Hencky strain smaller than 1 the shape of the geometries of the iPP and the LLDPE is almost the same. Correspondingly, the effective strain rate distributions over the mid-radius are similar for the three polymer melts, as shown in Fig. 13. The distributions are plotted at Hencky strains of $\varepsilon=0.5$, $\varepsilon=1, \varepsilon=2$ and $\varepsilon=3$, where the shear contributions decrease for increasing strains (see Fig. 12). So, although the polymer melts do not follow the LVE at relatively

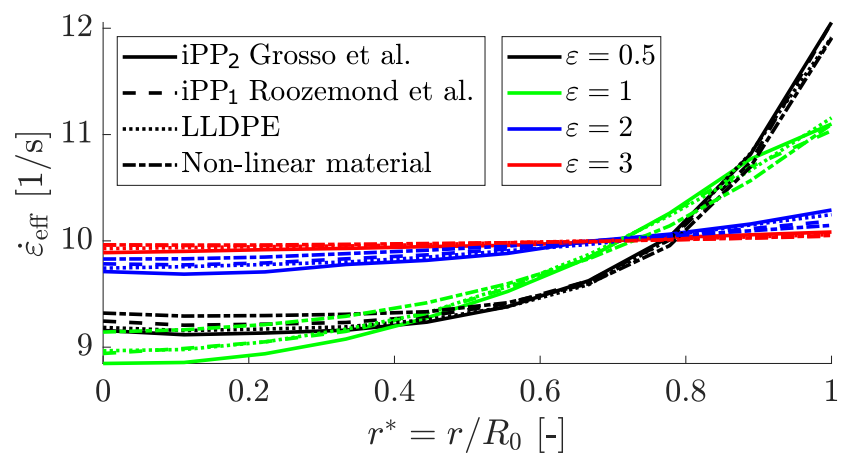

Fig. 13 Effective strain rate distributions for the iPPs, LLDPE and the arbitrary non-linear material at strains of $\varepsilon=0.5, \varepsilon=1, \varepsilon=2$ and $\varepsilon=3$. The filaments have a compressed aspect ratio of $\Lambda_{\mathrm{c}}=0.5$ and are extended with a strain rate of $\dot{\varepsilon}=10 \mathrm{~s}^{-1}$ at $T=160{ }^{\circ} \mathrm{C}$ small strains (i.e. show some non-linear behaviour at small strains), the mid-radius-based controller ensures that the strain rate distributions are approximately the same. Hence, geometrical effects dominate the non-linear effects of these materials.

Since the rheological behaviour of these materials is different, the similar effective strain rate distributions still result in different stress distributions, as shown in Fig. 14. Herein, the trace of the average conformation tensor is used as an indicator for stress. This stress value is normalized by dividing it by its mean value to compare the shape of the stress distributions. For increasing strain, the stress difference between the middle of the sample and the free surface increases. This is due to the distribution in strain rate over the mid-radius. For the LLDPE a more homogeneous stress distribution is observed at the highest strains $(\varepsilon=3)$. This is related to the strain hardening behaviour of this material. Because the strain hardening starts at a strain of 2 for LLDPE, where the strain rate distribution is nearly flat, the associated stress increase is nearly homogeneous over the mid-radius, and therefore the normalized stress distribution flattens. In case of the iPPs, there is no clear strain hardening behaviour present for strains smaller than 3. Hence, the non-homogeneous flow at small strains and the resulting stress distribution are still visible at larger strains. The stress distributions of these iPPs eventually flattens after strains of 3 , because of the homogeneous strain distribution, which results in a more homogeneous stress increase at the mid-radius.

Despite the different stress distributions over the midfilament radius for the various materials, Fig. 13 shows that the actual strain rate corresponds to the applied strain rate at a radial position $\mathrm{r} / \mathrm{R}$ around 0.72 . Similarly, at this location, the stress corresponds to the average stress of the relevant profile. Therefore, using the extensional viscosity

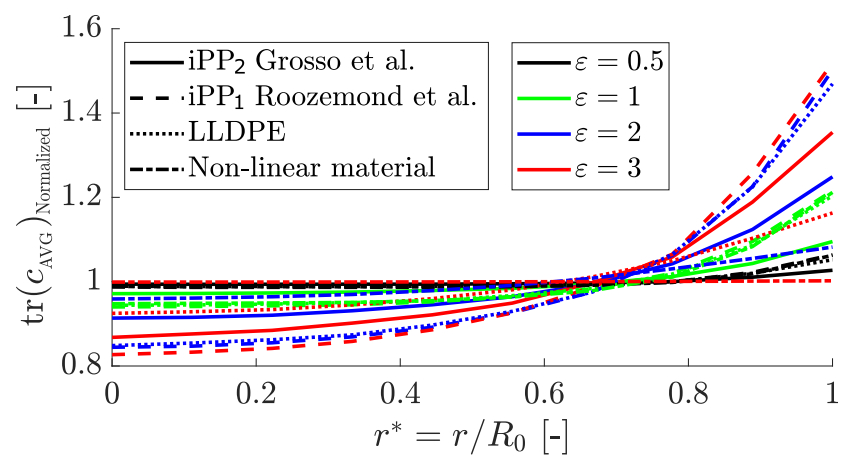

Fig. 14 Stress distributions for the iPPs, LLDPE and the arbitrary nonlinear material at strains of $\varepsilon=0.5, \varepsilon=1, \varepsilon=2$ and $\varepsilon=3$. Here, the normalized trace of the average conformation tensor is given, which gives an indication of the relative stress distributions. The compressed aspect ratio is $\Lambda_{\mathrm{c}}=0.5$ and the extension is performed at a strain rate of $\dot{\varepsilon}=10 \mathrm{~s}^{-1}$ at $T=160^{\circ} \mathrm{C}$ 
at the applied strain rate provides a consistent reference in the shear correction factor (Eq. 29) and causes its universal character. This occurrence of a fixed location at which applied and actual strain rate match, is similar to the behaviour of nonlinear materials in capillary flows (Schümmer and Worthoff 1978). Also there, deviations from the material-independent Schümmer correction factor occur in case of strongly nonlinear behaviour (Macosko 1994).

So from Fig. 13, it follows that at Hencky strains larger than 1 , the strain rate distribution over the mid-radius becomes flattened and converges to the applied strain rate of $\dot{\varepsilon}=10 \mathrm{~s}^{-1}$. Hence, if the non-linear effects before this strain do not significantly affect the geometry (as is the case for these polymer melts), then strain hardening after a strain of 1 would not affect the shear correction factor significantly. In case that the non-linear effects are larger at small strains, it is hypothesised that the shear correction factor in Eq. 41 is no longer valid. Strain hardening would then affect the shape of the geometry, because there is a strain rate distribution of about $10 \%$ at a strain of 1 , regardless of the material.

To test this hypothesis, an artificial non-linear material is introduced. The relaxation spectrum and non-linear parameters are given in Table 3. In Fig. 8d, it can be seen that a clear strain hardening at small strains is present for this artificial non-linear material. A simulation with this non-linear material at a strain rate of $\dot{\varepsilon}=10 \mathrm{~s}^{-1}$ results in a shear correction factor as shown in Fig. 12. Herein, the simulated shear correction factor does not match our proposed shear correction factor. Hence, the pronounced strain hardening at small strains affects the geometry and the strain rate and stress distributions over the mid-radius. In other words, the non-linear material's plate velocity needs to be higher to follow the ideal mid-radius with the controller in case of strain hardening. Note that for increasing compressed aspect ratio the deviation of the shear correction factor from the simulated shear correction factor becomes smaller. So for highly non-linear materials it is even more important to increase the initial aspect ratio, and therefore reduce the amount of correction needed.

At strains larger than 1.5, it can be seen in Fig. 12 that the shear correction factor is slightly larger than one. The reason for this is the conformation history which has not equilibrised yet. The history effects for the used strain rate of $10 \mathrm{~s}^{-1}$ will equilibrise at strains larger than 3. In Fig. 15, it can be seen that for a strain rate of $\dot{\varepsilon}=1.71 \mathrm{~s}^{-1}$ this equilibration takes place at relatively smaller strains. This is because there is more time for the polymer to equilibrise. Note that even for this highly non-linear material no strain rate dependency is observed for small enough strains.

For a wide range of polymer melts and solutions, and even more complex polymeric materials such as ionomers

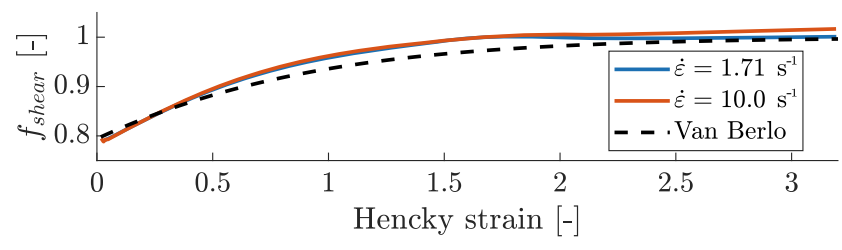

Fig. 15 Shear correction factor of the arbitrary non-linear material for strain rates of $\dot{\varepsilon}=1.71 \mathrm{~s}^{-1}$ and $\dot{\varepsilon}=10 \mathrm{~s}^{-1}$. The compressed aspect ratio is $\Lambda_{\mathrm{c}}=0.5$ and the extension is performed at $T=160{ }^{\circ} \mathrm{C}$

and transient polymer networks pronounced deviations from linear viscoelasticity (such as strain hardening) only start to appear at strains larger than one (McKinley and Sridhar 2002; Costanzo et al. 2016; Shabbir et al. 2017; Arora et al. 2017; Münstedt 2018). Therefore, it is possible to use the proposed shear correction factor in Eq. 41 in a wide range of FiSER experiments. However, for specific materials, this highly non-linear behaviour in extensional flow can be observed at strains much smaller than one, as for instance for gluten gels, supramolecular polymers and glass fiberfilled polymers (Ng and McKinley 2008; Férec et al. 2009; Cui et al. 2018). For these cases, care should be taken since the proposed shear correction factor for filament stretching rheometry is no longer valid, as shown in the case of the artificial non-linear material. However, for an increased initial aspect ratio, the shear contributions decrease and the flow becomes more homogeneous. Therefore, the strain rate distributions over the mid-radius will be flattened and converge to the applied strain rate at smaller strains. This implies that a near locally homogeneous flow can be achieved before the start of the strain hardening of the non-linear material. As a result, the simulated shear correction factor converges towards the proposed universal shear correction factor, as shown in Fig. 12.

\section{Conclusions}

A complete numerical tool, reproducing the flow and resulting forces (rheology) of two iPPs, a LLDPE and an artificial non-linear material in a FiSER, is presented. In particular, finite element simulations are used that simultaneously solve the mass balance and the momentum balance using a non-linear viscoelastic constitutive equation (XPP model). Also, a controller is added to mimic the locally controlled uniaxial extensional flow in the FiSER. The simulations are validated by comparing force results with experiments and analytical predictions. The typical force overshoot at the start of a filament stretching experiment, caused by shear flow near the no-slip boundaries of the pistons, is also present in the simulations. Therefore, it is possible to investigate the shear effects for 
non-linear viscoelastic materials. Existing shear correction factors perform well for sufficiently high $\Lambda_{c}$ values, even in the non-linear viscoelastic regime. To correct for shear effects at small $\Lambda_{c}$ values, an empirical correlation for the non-linear shear correction factor is presented. This shear correction factor shows good agreement with the simulations of the polymer melts and has been validated for multiple materials at different strain rates and initial aspect ratios. For a wide range of polymer melts and solutions, and even more complex polymeric materials such as ionomers and transient polymer networks, which show moderate nonlinear behaviour at strains smaller than one, it is possible to use the universal shear correction factor proposed in this study. However, in specific materials such as gluten gels, supramolecular polymers and glass fiber-filled polymers which show highly non-linear behaviour at strains smaller than one, the universal shear correction factor should be used with care. For these materials, it is important to use an initial geometry which ensures a near homogeneous purely uniaxial flow before the start of the highly nonlinear behaviour of the material. This can be achieved by increasing the initial aspect ratio. In future research it is interesting to investigate how the shear correction factor depends on the constitutive behaviour of these highly nonlinear materials.

Acknowledgements The authors thank Dr. M.A. Hulsen at the Eindhoven University of Technology (TU/e), Eindhoven, the Netherlands for providing access to the Toolkit for Finite Element Method (TFEM) software libraries.

\section{Declarations}

Conflict of interest The authors declare no competing interests.

Open Access This article is licensed under a Creative Commons Attribution 4.0 International License, which permits use, sharing, adaptation, distribution and reproduction in any medium or format, as long as you give appropriate credit to the original author(s) and the source, provide a link to the Creative Commons licence, and indicate if changes were made. The images or other third party material in this article are included in the article's Creative Commons licence, unless indicated otherwise in a credit line to the material. If material is not included in the article's Creative Commons licence and your intended use is not permitted by statutory regulation or exceeds the permitted use, you will need to obtain permission directly from the copyright holder. To view a copy of this licence, visit http://creativecommons. org/licenses/by/4.0/.

\section{References}

Anna SL, Rogers C, McKinley GH (1999) On controlling the kinematics of a filament stretching rheometer using using a realtime active control mechanism. J Non-Newtonian Fluid Mech 87:307-335. https://doi.org/10.1016/S0377-0257(99)00072-5
Anna SL, McKinley GH, Nguyen DA, Sridhar T, Muller SJ, Huang J, James DF (2001) An interlaboratory comparison of measurements from filament-stretching rheometers using common test fluids. J Rheol 45(1):83-114. https://doi.org/10.1122/1.1332388

Arora S, Shabbir A, Hassager O, Ligoure C, Ramos L (2017) Brittle fracture of polymer transient networks. J Rheol 61(6):1267-1275. https://doi.org/10.1122/1.4997587

Bach A, Rasmussen HK, Hassager O (2003) Extensional viscosity for polymer melts measured in the filament stretching rheometer. $\mathrm{J}$ Rheol 47(2):429-441. https://doi.org/10.1122/1.1545072

Baltussen MGHM, Hulsen MA, Peters GWM (2010) Numerical simulation of the fountain flow instability in injection molding. J Non-Newtonian Fluid Mech 165(11):631-640. https://doi.org/10.1016/j.jnnfm.2010.03.001

Berg S, Kröger R, Rath HJ (1994) Measurement of extensional viscosity by stretching large liquid bridges in microgravity. J Non-Newtonian Fluid Mech 55(3):307-319. https://doi.org/10.1016/0377-0257(94)80075-8

van Berlo FPA, Cardinaels R, Peters GWM, Anderson PD (2020) A numerical study of extensional flow-induced crystallization in filament stretching rheometry. Polym Crystallization. https://doi.org/10.1002/pcr2.10154

Bird R, Armstrong R, Hassager O (1987) Dynamics of polymeric liquids, Volume 1: Fluid mechanics, 2nd edn. Wiley, New York

Bird RB (1976) Useful non-newtonian models. Annu Rev Fluid Mech 8(1):13-34. https://doi.org/10.1146/annurev.fl.08.010176.000305

Bogaerds ACB, Grillet AM, Peters GWM, Baaijens FPT (2002) Stability analysis of polymer shear flows using the extended pom-pom constitutive equations. J Non-Newtonian Fluid Mech 108(1):187-208. https://doi.org/10.1016/S0377-0257(02)00130-1

Brooks AN, Hughes TJ (1982) Streamline upwind/petrov-galerkin formulations for convection dominated flows with particular emphasis on the incompressible navier-stokes equations. Comput Methods Appl Mech Eng 32(1):199-259. https://doi.org/10.1016/0045-7825(82)90071-8

Chellamuthu M, Arora D, Winter HH, Rothstein JP (2011) Extensional flow-induced crystallization of isotactic poly-1-butene using a filament stretching rheometer. J Rheol 55(4):901-920. https://doi.org/10.1122/1.3593471

Cogswell FN (1972) Converging flow of polymer melts in extrusion dies. Polym Eng Sci 12(1):64-73. https://doi.org/10.1002/pen.760120111

Costanzo S, Huang Q, Ianniruberto G, Marrucci G, Hassager O, Vlassopoulos D (2016) Shear and extensional rheology of polystyrene melts and solutions with the same number of entanglements. Macromolecules 49(10):3925-3935. https://doi.org/10.1021/acs.macromol.6b00409

Cui G, Boudara VAH, Huang Q, Baeza GP, Wilson AJ, Hassager O, Read DJ, Mattsson J (2018) Linear shear and nonlinear extensional rheology of unentangled supramolecular side-chain polymers. J Rheol 62(5):1155-1174. https://doi.org/10.1122/1.5012349

D‘Avino G, Hulsen MA, Maffettone PL (2012) Decoupled transient schemes for viscoelastic fluid flow with inertia. Comput Fluids 66:183-193. https://doi.org/10.1016/j.compfluid.2012.06.023

van Drongelen M, Roozemond PC, Troisi EM, Doufas AK, Peters GWM (2015) Characterization of the primary and secondary crystallization kinetics of a linear low-density polyethylene in quiescent- and flow-conditions. Polymer 76:254-270. https://doi.org/10.1016/j.polymer.2015.09.010

van Erp TB, Roozemond PC, Peters GWM (2013) Flow-enhanced crystallization kinetics of ipp during cooling at elevated pressure: Characterization, validation, and development. Macromol Theory Simul 22(5):309-318. https://doi.org/10.1002/mats.201300004

Férec J, Heuzey MC, Pérez-González J, de Vargas L, Ausias G, Carreau PJ (2009) Investigation of the rheological properties of 
short glass fiber-filled polypropylene in extensional flow. Rheol Acta 48(1):59-72. https://doi.org/10.1007/s00397-008-0309-9

Fuller GG, Cathey CA, Hubbard B, Zebrowski BE (1987) Extensional viscosity measurements for low-viscosity fluids. J Rheol 31(3):235-249. https://doi.org/10.1122/1.549923

Geuzaine C, Remacle JF (2009) Gmsh: A 3-d finite element mesh generator with built-in pre- and post-processing facilities. Int $\mathbf{J}$ Numer Methods Eng 79(11):1309-1331. https://doi.org/10.1002/nme.2579

Grosso G, Troisi EM, Jaensson NO, Anderson PD, Peters GWM (2019) Modelling of flow induced crystallization of ipp: multiple phases and multiple morphologies. Polymer :121806. https://doi.org/10.1016/j.polymer.2019.121806

Hassager O, Marin JMR, Yu K, Rasmussen HK (2010) Polymeric liquids in extension: fluid mechanics or rheometry? Rheol Acta 49(6):543-554. https://doi.org/10.1007/s00397-010-0444-y

Hirt C, Amsden A, Cook J (1974) An arbitrary lagrangian-eulerian computing method for all flow speeds. J Comput Phys 14(3):227253. https://doi.org/10.1016/0021-9991(74)90051-5

Hodder P, Franck A (2005) A new tool for measuring extensional viscosity. Proc Nord Rheol Soc 13:227-232

Housmans JW, Gahleitner M, Peters GWM, Meijer HEH (2009) Structure-property relations in molded, nucleated isotactic polypropylene. Polymer 50(10):2304-2319. https://doi.org/10.1016/j.polymer.2009.02.050

Huang Q, Rasmussen HK, Skov AL, Hassager O (2012) Stress relaxation and reversed flow of low-density polyethylene melts following uniaxial extension. J Rheol 56(6):1535-1554. https://doi.org/10.1122/1.4752759

Huang Q, Mangnus M, Alvarez NJ, Koopmans R, Hassager O (2016) A new look at extensional rheology of low-density polyethylene. Rheol Acta 55(5):343-350. https://doi-org.dianus.libr.tue.nl/10. 1007/s00397-016-0921-z

Hulsen MA, Fattal R, Kupferman R (2005) Flow of viscoelastic fluids past a cylinder at high weissenberg number: stabilized simulations using matrix logarithms. J Non-Newtonian Fluid Mech 127(1):2739. https://doi.org/10.1016/j.jnnfm.2005.01.002

Jaensson NO, Hulsen MA, Anderson PD (2015) Stokes-cahnhilliard formulations and simulations of two-phase flows with suspended rigid particles. Comput Fluids 111:1-17. https://doi.org/10.1016/j.compfluid.2014.12.023

Kase S, Matsuo $\mathrm{T}$ (1965) Studies on melt spinning. i. fundamental equations on the dynamics of melt spinning. J Polym Sci Part A Gen Pap 3(7):2541-2554. https://doi.org/10.1002/pol.1965.100030712

Kolte MI, Rasmussen HK, Hassager O (1997) Transient filament stretching rheometer. Rheol Acta 36(3):285-302. https://doi.org/10.1007/BF00366670

Kröger R, Berg S, Delgado A, Rath HJ (1992) Stretching behaviour of large polymeric and newtonian liquid bridges in plateau simulation. J Non-Newtonian Fluid Mech 45(3):385-400. https://doi.org/10.1016/0377-0257(92)80069-A

Macosko CW (1994) Rheology principles. Measu Appl

Marín JMR, Huusom JK, Alvarez NJ, Huang Q, Rasmussen HK, Bach A, Skov AL, Hassager O (2013) A control scheme for filament stretching rheometers with application to polymer melts. J Non-Newtonian Fluid Mech 194:14-22. https://doi.org/10.1016/j.jnnfm.2012.10.007

Matta JE, Tytus RP (1990) Liquid stretching using a falling cylinder. J Non-Newtonian Fluid Mech 35(2):215-229. https://doi.org/10.1016/0377-0257(90)85050-9

McKinley GH, Sridhar T (2002) Filament-stretching rheometry of complex fluids. Annu Rev Fluid Mech 34(1):375-415. https://doi.org/10.1146/annurev.fluid.34.083001.125207
McKinley GH, Anna SL, Tripathi A, Yao M (1999) Extensional rheometry of polymeric fluids and the uniaxial elongation of viscoelastic filaments. Proceedings of the 15th international polymer processing society. Netherlands 83

Meissner J, Raible T, Stephenson SE (1981) Rotary clamp in uniaxial and biaxial extensional rheometry of polymer melts. J Rheol 25(6):673-674. https://doi.org/10.1122/1.549635

Münstedt H (1975) Viscoelasticity of polystyrene melts in tensile creep experiments. Rheol Acta 14(12):1077-1088. https://doi.org/10.1007/BF01515903

Münstedt H (2018) Extensional rheology and processing of polymeric materials. Int Polym Process 33(5):594-618. https://doi.org/10.3139/217.3532

Morrison FA (2001) Understanding rheology. Oxford University Press, New York

Ng TSK, McKinley GH (2008) Power law gels at finite strains: The nonlinear rheology of gluten gels. J Rheol 52(2):417-449. https://doi.org/10.1122/1.2828018

Nielsen JK, Rasmussen HK, Hassager O (2008) Stress relaxation of narrow molar mass distribution polystyrene following uniaxial extension. $\mathrm{J}$ Rheol 52(4):885-899. https://doi.org/10.1122/1.2930872

Nielsen JK, Hassager O, Rasmussen HK, McKinley GH (2009) Observing the chain stretch transition in a highly entangled polyisoprene melt using transient extensional rheometry. J Rheol 53(6):1327-1346. https://sor.scitation.org/doi/full/10.1122/ 1.3208073

Ooi YW, Sridhar T (1994) Extensional rheometry of fluid s1. J Non-Newtonian Fluid Mech 52(2):153-162. https://doi.org/10.1016/0377-0257(94)80047-2

Orr N, Sridhar T (1999) Probing the dynamics of polymer solutions in extensional flow using step strain rate experiments. J Non-Newtonian Fluid Mech 82(2):203-232. https://doi.org/10.1016/S0377-0257(98)00163-3

Pepe J, Cleven LC, Suijkerbuijk EJMC, Dekkers ECA, HermidaMerino D, Cardinaels R, Peters GWM, Anderson PD (2020) A filament stretching rheometer for in situ $\mathrm{x}$-ray experiments: combining rheology and crystalline morphology characterization. Rev Sci Instrum 91(7):073903. https://doi.org/10.1063/5.0008224

Rasmussen HK, Bejenariu AG, Hassager O, Auhl D (2010) Experimental evaluation of the pure configurational stress assumption in the flow dynamics of entangled polymer melts. J Rheol 54(6):1325-1336. https://doi.org/10.1122/1.3496378

Roozemond PC, van Drongelen M, Ma Z, Hulsen MA, Peters GWM (2015) Modeling flow-induced crystallization in isotactic polypropylene at high shear rates. J Rheol 59(3):613-642. https://doi.org/10.1122/1.4913696

Schümmer P, Worthoff R (1978) An elementary method for the evaluation of a flow curve. Chem Eng Sci 33(6):759-763. https://doi.org/10.1016/0009-2509(78)80054-2

Sentmanat M (2003) A novel device for characterizing polymer flows in uniaxial extension. In: Annual technical conference - ANTEC conference proceedings, vol 1, pp 992-996

Shabbir A, Huang Q, Baeza GP, Vlassopoulos D, Chen Q, Colby RH, Alvarez NJ, Hassager O (2017) Nonlinear shear and uniaxial extensional rheology of polyether-estersulfonate copolymer ionomer melts. J Rheol 61(6):1279-1289. https://doi.org/10.1122/1.4998158

Spiegelberg S, Ables DC, Mckinley G (1996) The role of endeffects on measurements of extensional viscosity in filament stretching rheometers. J Non-Newtonian Fluid Mech 64:229-267. https://doi.org/10.1016/0377-0257(96)01439-5

Sridhar T, Tirtaatmadja V, Nguyen D, Gupta R (1991) Measurement of extensional viscosity of polymer solutions. J Non-Newtonian 
Fluid Mech 40(3):271-280. http://doi.org/10.1016/0377-0257(91) 87012-M

Szabo P (1997) Transient filament stretching rheometer. Rheol Acta 36(3):277-284. https://doi.org/10.1007/BF00366669

Tanner RI (2000) Engineering rheology. Oxford Engineering Science Series, OUP Oxford

Tirtaatmadja V, Sridhar T (1993) A filament stretching device for measurement of extensional viscosity. J Rheol 37:1081-1102. https://doi.org/10.1122/1.550372

Verbeeten WMH, Peters GWM, Baaijens FPT (2001) Differential constitutive equations for polymer melts: the extended pom-pom model. J Rheol 45(4):823-843. https://doi.org/10.1122/1.1380426

Verbeeten WMH, Peters GWM, Baaijens FPT (2004) Numerical simulations of the planar contraction flow for a polyethylene melt using the xpp model. J Non-Newtonian Fluid Mech 117(2):73-84. https://doi.org/10.1016/j.jnnfm.2003.12.003
Vinogradov GV, Radushkevich BV, Fikhman VD (1970) Extension of elastic liquids: polyisobutylene. J Polym Sci Part A-2: Polym Phys 8(1):1-17. https://doi.org/10.1002/pol.1970.160080101

Yao M, McKinley GH (1998) Numerical simulation of extensional deformations of viscoelastic liquid bridges in filament stretching devices. J Non-Newtonian Fluid Mech 74(1):47-88. https://doi.org/10.1016/S0377-0257(97)00052-9

Zhang XM, Li H, Chen WX, Feng LF (2012) Rheological properties and morphological evolutions of polypropylene/ethylenebutene copolymer blends. Polym Eng Sci 52(8):1740-1748. https://doi.org/10.1002/pen.23116

Publisher's note Springer Nature remains neutral with regard to jurisdictional claims in published maps and institutional affiliations. 